TRANSACTIONS OF THE

AMERICAN MATHEMATICAL SOCIETY

Volume 349, Number 2, February 1997, Pages 481-527

S 0002-9947(97)01616-4

\title{
COHERENT FUNCTORS, WITH APPLICATION TO TORSION IN THE PICARD GROUP
}

\author{
DAVID B. JAFFE
}

\begin{abstract}
Let $A$ be a commutative noetherian ring. We investigate a class of functors from $\langle\langle$ commutative $A$-algebras $\rangle\rangle$ to $\langle\langle$ sets $\rangle\rangle$, which we call coherent. When such a functor $F$ in fact takes its values in $\langle\langle$ abelian groups $\rangle\rangle$, we show that there are only finitely many prime numbers $p$ such that ${ }_{p} F(A)$ is infinite, and that none of these primes are invertible in $A$. This (and related statements) yield information about torsion in $\operatorname{Pic}(A)$. For example, if $A$ is of finite type over $\mathbb{Z}$, we prove that the torsion in $\operatorname{Pic}(A)$ is supported at a finite set of primes, and if ${ }_{p} \operatorname{Pic}(A)$ is infinite, then the prime $p$ is not invertible in $A$. These results use the (already known) fact that if such an $A$ is normal, then $\operatorname{Pic}(A)$ is finitely generated. We obtain a parallel result for a reduced scheme $X$ of finite type over $\mathbb{Z}$. We classify the groups which can occur as the Picard group of a scheme of finite type over a finite field.
\end{abstract}

\section{INTRODUCTION}

Coherent functors (introductory remarks). Let us say that an A-functor is a functor from the category of commutative $A$-algebras to $\langle\langle$ sets $\rangle\rangle$. Some such $A$ functors have additional structure: they are actually functors from $\langle\langle$ commutative $A$-algebras $\rangle\rangle$ to $\langle\langle$ groups $\rangle\rangle$. We refer to such functors as group-valued $A$-functors. We will also consider $A$-functors $F$ such that $F(B)$ is a $B$-module for every $B$; these module-valued $A$-functors are discussed later in the Introduction. For now, all $A$-functors which we consider will be treated as set-valued functors.

An $A$-functor is coherent if it may be built up as an iterated finite limit of functors of the form $\underline{M}$, given by $\underline{M}(B)=M \otimes_{A} B$, where $M$ is a finitely generated $A$-module. We do not know if every coherent functor may be expressed as a finite limit of such functors $\underline{M}$. However, the analogous question regarding module-valued functors is answered affirmatively below.

The idea of coherent functor was originally devised by Auslander [5], in a somewhat different setting; his notion of coherence applied to functors from an abelian category to $\langle\langle$ abelian groups $\rangle\rangle$. Later Artin [2] transposed Auslander's notion to a setting closer to that given here. Artin also raised a question about coherence of higher direct images as functors. This question is considered in an Appendix.

If an $A$-functor is representable by a commutative $A$-algebra of finite type, then it is coherent. There are many examples of nonrepresentable $A$-functors which are coherent. For example, if $M$ is a finitely generated $A$-module, then $B \mapsto$

Received by the editors July 1, 1994 and, in revised form, September 19, 1995.

1991 Mathematics Subject Classification. Primary 14C22, 18A25, 14K30, 18A40.

Key words and phrases. Coherent functor, representable functor, Picard group.

Partially supported by the National Science Foundation. 
$\operatorname{Aut}_{B-\bmod }\left(M \otimes_{A} B\right)$ defines a coherent $A$-functor. More examples may be found in $\S 4$.

A module-valued $A$-functor is an (abelian group)-valued $A$-functor $F$, together with the following additional structure: for each commutative $A$-algebra $B, F(B)$ has the structure of a $B$-module, such that for any homomorphism $B_{1} \rightarrow B_{2}$ of commutative $A$-algebras, the induced map $F\left(B_{1}\right) \rightarrow F\left(B_{2}\right)$ is a homomorphism of $B_{1}$-modules. The module-valued $A$-functors form an abelian category.

A module-valued $A$-functor $F$ is module-coherent if there exists a homomorphism $f: M \rightarrow N$ of finitely generated $A$-modules such that $F$ is isomorphic to the module-valued $A$-functor given by $B \mapsto \operatorname{ker}\left(f \otimes_{A} B\right)$. The module-coherent $A$ functors form a full subcategory of the category of module-valued $A$-functors.

Most examples of module-valued $A$-functors are induced naturally by functors from $\langle\langle A$-modules $\rangle\rangle$ to $\langle\langle A$-modules $\rangle\rangle$. Certainly for many purposes it makes more sense to study the latter sort of functor. On the other hand, as pointed out by Artin [2], one can set up a correspondence between module-coherent $A$-functors and functors from $\langle\langle A$-modules $\rangle\rangle$ to $\langle\langle A$-modules $\rangle\rangle$ which satisfy an analogous coherence axiom. This creates a bridge to the ideas of Auslander [5] and Grothendieck ([17], $\S 7)$. We have not exploited this point of view.

A key result is that if $\sigma: F \rightarrow G$ is a morphism of module-coherent $A$-functors, then $\operatorname{ker}(\sigma)$ and $\operatorname{coker}(\sigma)$ are module-coherent. (These are to be computed in $\langle\langle$ module-valued $A$-functors $\rangle\rangle$.) One deduces easily from this that any finite limit or finite colimit of module-coherent $A$-functors is module-coherent. In particular, the iterated finite limit construction which we used in the definition of coherent $A$ functor is not necessary here, although in the body of the paper we find it convenient to begin with a definition of module-coherent which uses iterated finite limits.

Let $X$ be a noetherian scheme. An $X$-functor is a functor from $\langle\langle X \text {-schemes }\rangle\rangle^{\circ}$ to $\langle\langle$ sets $\rangle\rangle$. We define the notion of coherent $X$-functor by analogy with the definition for $A$-functors. When $X=\operatorname{Spec}(A)$, the theory of coherent $X$-functors is identical to the theory of coherent $A$-functors. Similarly, we define module-coherent $X$ functors. We show that the property of being a module-coherent $X$-functor is local on $X$, assuming that $X$ is separated. We conjecture that the property of being a coherent $X$-functor is local on $X$.

Finiteness theorems (introductory remarks). Let $F$ be an (abelian group)valued coherent $X$-functor. We discuss finiteness results for $F(X)$. These translate (but rarely verbatim) to finiteness results for $\operatorname{Pic}(X)$.

We prove that there are only finitely many primes $p$ such that ${ }_{p} F(X)$ is infinite, and that none of these primes are invertible in $\Gamma\left(X, \mathcal{O}_{X}\right)$. A stronger form of this statement holds if $X$ is essentially of finite type over $\mathbb{Z}$ or over $\mathbb{Z}_{p}$ for some prime $p$. For example, if $X$ is of finite type over $\mathbb{Q}$ or over $\mathbb{Q}_{p}$, then the torsion subgroup of $F(X)$ is finite.

Assuming that $X$ is reduced and that the canonical map $X_{\text {nor }} \rightarrow X$ is finite, consider the quotient sheaf $F=\mathcal{O}_{X_{\text {nor }}}^{*} / \mathcal{O}_{X}^{*}$ on $X$. We extend $F$ to an (abelian group)-valued $X$-functor, also denoted here by $F$. We do not know if $F$ is coherent, but we are able (more or less) to find an (abelian group)-valued coherent $X$-functor $G$, and a morphism $\psi: F \rightarrow G$ such that $\psi(X)$ is injective. We say "more or less" because the actual proof works via a sequence of partial normalizations. The end result however is the same: there are only finitely many primes $p$ such that ${ }_{p} F(X)$ 
is infinite, and none of these primes are invertible in $\Gamma\left(X, \mathcal{O}_{X}\right)$. There is also a stronger form for certain $X$ as discussed in the previous paragraph.

Let $Q$ be the group $\Gamma\left(\mathcal{O}_{X_{\text {nor }}}^{*}\right) / \Gamma\left(\mathcal{O}_{X}^{*}\right)$, and let $K=\operatorname{Ker}\left[\operatorname{Pic}(X) \rightarrow \operatorname{Pic}\left(X_{\text {nor }}\right)\right]$. Then there is a short exact sequence

$$
0 \rightarrow Q \rightarrow F(X) \rightarrow K \rightarrow 0 .
$$

It follows that if $Q$ is finitely generated, then there are only finitely many primes $p$ such that ${ }_{p} K$ is infinite, and none of these primes are invertible in $\Gamma\left(X, \mathcal{O}_{X}\right)$. This holds for instance if $X$ is of finite type over $\mathbb{Z}$, thereby yielding one of the results stated in the abstract.

When $X$ is of finite type over a field $k$, it has been shown [20] that ${ }_{n} \operatorname{Pic}(X)$ is finite for every $n$ which is invertible in $k$. For a finite field $k$ of characteristic $p$, we prove a strengthened form of this statement: modulo $p$-power torsion, $\operatorname{Pic}(X)$ is finitely generated. We completely describe the structure of $\operatorname{Pic}(X)$ as an abstract abelian group.

The following related result is relevant. Claborn [11] has shown that for every abelian group, there exists a Dedekind domain containing $\mathbb{Q}$, whose Picard group is the given abelian group. (See also [14], §14.) In particular, for suitable $X, \operatorname{Pic}(X)$ itself has infinite $n$-torsion, for every $n$.

It would be interesting to know to what extent the results of this paper on $\operatorname{Pic}(X)$ can be obtained via étale cohomology.

Acknowledgements. I thank Deligne and Ogus for much help on the Appendix.

Conventions. We agree on the following conventions:

- $A$ denotes an arbitrary commutative noetherian ring (unless specified otherwise); $B$ usually denotes an arbitrary commutative $A$-algebra.

- $X$ denotes an arbitrary noetherian scheme.

- By a Zariski sheaf, we mean a sheaf for the Zariski topology.

- If $S, T$ are sets, and $M$ is an abelian group, by a left exact sequence

$$
S \longmapsto T \stackrel{f}{\rightarrow} M
$$

we mean that the map from $S$ to $T$ is injective, and that $S$ is the kernel of the map from $T$ to $M$, meaning that $S=\{t \in T: f(t)=0\}$. Similar language applies when $S, T$, and $M$ are functors.

Summary of some definitions. For the convenience of the reader, we summarize here most of the types of functors which appear in the paper. This is intended as a rough reference, and not a source of precise definitions.

An $A$-functor is a functor from commutative $A$-algebras to sets. We are primarily interested in the quasi-coherent ones, which are built up from $A$-functors of the form $\bar{M}$, where $M$ is an arbitrary $A$-module. If they are built up using module maps (and not just set maps), then they are a species of module-valued A-functor, called module-quasi-coherent. If one only uses finitely generated $A$-modules, then one gets coherent $A$-functors (or module-coherent $A$-functors, if one uses module maps). A functor of the form $\bar{M}$ is strictly module-quasi-coherent, and is strictly module-coherent if $M$ is finitely generated. A functor of the form $\operatorname{Ker}\left[\overline{A^{n}} \rightarrow \overline{A^{k}}\right]$ is linearly representable. Similarly one obtains linearly quasi-representable A-functors 
by allowing arbitrary free $A$-modules instead of $A^{n}, A^{k}$. A bar-module-coherent $A$ functor is a module-quasi-coherent $A$-functor which is the epimorphic image of a module-coherent $A$-functor.

An $X$-functor is a contravariant functor from $X$-schemes to sets. All of the above definitions carry over to $X$-functors. Each type has moreover a local version, which corresponds to requiring that the given property hold locally on $X$.

Any sort of $A$-functor or $X$-functor defined above (and which is not intrinsically module-valued) has a group-valued version; this just means that the functor (a priori set-valued) factors though the forgetful functor from groups to sets.

Guide to sections of the paper. In $\S 1$, we define coherent $A$-functors. In $\S 2$, we define module-coherent $A$-functors and show that they all come from homomorphisms of finitely generated $A$-modules. Section 3 is parallel to $\S 1$ and $\S 2$, but we consider arbitrary modules, thereby arriving at the theory of quasi-coherent and module-quasi-coherent $A$-functors. Section 4 contains examples. Section 5 contains the theory of $X$-functors. In $\S 6$, we consider continuity, i.e. the extent to which functors preserve limits. Sections 7 and 8 give finiteness results for global sections of $X$-functors. Section 9 contains applications to the Picard group. As an Appendix, we consider an old problem of Artin, regarding whether higher direct images preserve coherence.

\section{Coherent Functors}

In this section we develop the basic theory of coherent functors. We have already defined the notion of $A$-functor in the Introduction. These form a category $\langle\langle A-$ functors $\rangle\rangle$ whose morphisms are natural transformations.

If $M$ is an $A$-module, then there is an $A$-functor $\underline{M}$ given by $\underline{M}(B)=M \otimes_{A} B$. If a given $A$-functor $F$ is isomorphic to $\underline{M}$ for some finitely generated $A$-module $M$, we shall say that $F$ is strictly coherent.

Definition. Let $\mathcal{C}$ be a category. Let $S$ be a collection of objects in $\mathcal{C}$. Let $S_{0}=S$, and for each $n \geq 0$, let $S_{n+1}$ be the collection of all objects of $\mathcal{C}$ which may be obtained as limits (in $\mathcal{C}$ ) of diagrams involving finitely many objects in $S_{n}$ and finitely many morphisms. Let $S_{\infty}=\bigcup_{n=0}^{\infty} S_{n}$. Then we say that the objects in $S_{\infty}$ are iterated finite limits of objects in $S$.

Definition. An $A$-functor is coherent if it may be obtained as an iterated finite limit of strictly coherent $A$-functors, where the limits are taken in $\langle\langle A$-functors $\rangle\rangle$.

We may define $\langle\langle$ coherent $A$-functors $\rangle\rangle$ : it is a full subcategory of $\langle\langle A$-functors $\rangle\rangle$, which may be thought of as the finite completion of the subcategory $\langle\langle$ strictly coherent $A$-functors $\rangle\rangle$ of $\langle\langle A$-functors $\rangle\rangle$.

Let $\mathcal{C}_{A}^{0}$ denote the collection of strictly coherent $A$-functors. For each $n \geq 0$, let $\mathcal{C}_{A}^{n+1}$ denote the collection of $A$-functors which may be obtained as finite limits (inside $\langle\langle A$-functors $\rangle\rangle)$ of objects in $\mathcal{C}_{A}^{n}$. We have

$$
\mathcal{C}_{A}^{0} \subset \mathcal{C}_{A}^{1} \subset \mathcal{C}_{A}^{2} \subset \cdots \text {. }
$$

Let $\mathcal{C}_{A}=\bigcup_{n=0}^{\infty} \mathcal{C}_{A}^{n}$. Then the objects in $\mathcal{C}_{A}$ are exactly the coherent $A$-functors.

Definition. Let $F$ be a coherent $A$-functor. Then the level of $F$ is the smallest integer $n$ such that $F \in \mathcal{C}_{A}^{n}$. 
In some proofs, we will need to induct on the level of a given coherent $A$-functor. This process will be facilitated by the following lemma, whose proof is left to the reader.

Lemma 1.1. Let $F$ be a coherent A-functor of level $n \geq 1$. Then there exists a left exact sequence

$$
F \longmapsto G \rightarrow \underline{M}
$$

in which $G$ is a coherent A-functor of level $n-1$ and $M$ is a finitely generated A-module. Moreover, $F$ may be embedded as a subfunctor of a strictly coherent A-functor.

If $k$ is a field, then every coherent $k$-functor is representable, and from this one deduces that every coherent $k$-functor has level $\leq 1$. Later $(2.9)$ we shall prove that a large class of coherent $k$-functors have level $\leq 1$. However, we do not know the answer to the following basic question:

Problem 1.2. Does every coherent $A$-functor have level $\leq 1$ ?

Note that for a given $A$-functor $F$, this is the case if and only if there exist finitely generated $A$-modules $M$ and $N$, together with a morphism $\phi: \underline{M} \rightarrow \underline{N}$ of $A$-functors such that $F$ is the "kernel" of $\phi$, meaning that

$$
F(B)=\left\{x \in M \otimes_{A} B: \phi(x)=0\right\} .
$$

The maps $\phi(B)$ need not be homomorphisms of $B$-modules.

In some situations, for a given coherent $A$-functor $F$, it will be necessary to consider the set $\mathcal{S}=\left\{M_{1}, \ldots, M_{n}\right\}$ of all $A$-modules which enter into its construction. This set is not uniquely determined by $F$. Also it does not carry information about multiplicity: $\mathcal{S}$ might consist of a single module $M$, but many copies of $M$ might enter into the construction of $F$. If $F$ has level 0 , we can choose $\mathcal{S}$ to have one element. If $F$ has level 1 , then we may view $F$ as a limit of strictly coherent $A$-functors, and thus we may choose $\mathcal{S}$ to consist of the corresponding modules. If $F$ has level 2 , then $F$ is a limit of level 1 coherent $A$-functors $F_{1}, \ldots, F_{k}$, and we may choose $\mathcal{S}$ to be the union of the sets corresponding (as just considered) to $F_{1}, \ldots, F_{k}$. In any case, we shall say that $F$ is built up from $M_{1}, \ldots, M_{n}$. Conversely, given an arbitrary class $\mathcal{S}$ of finitely generated $A$-modules, we may speak of coherent $A$-functors which are built up from $\mathcal{S}$, meaning that such $A$-functors are built up from finite subsets of $\mathcal{S}$.

\section{Module-COHEREnT FunCtORS}

In this section we develop the basic theory of module-coherent functors. The definition given initially will not be the same as that given in the Introduction. It is only after considerable work that we will find (2.9) that the two definitions agree.

We have already defined the notion of module-valued $A$-functor in the Introduction. If $F$ and $G$ are module-valued $A$-functors, then a morphism $\sigma: F \rightarrow G$ is a natural transformation of functors, in the following sense. It is a system of homomorphisms $\sigma(B): F(B) \rightarrow G(B)$ of $B$-modules, for each commutative $A$-algebra 
$B$, such that for any $A$-algebra homomorphism $f: B_{1} \rightarrow B_{2}$, the diagram

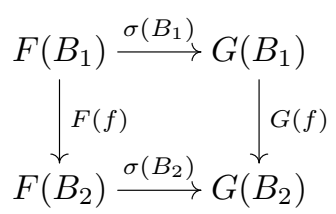

commutes.

With this definition of morphism, the module-valued $A$-functors form a category, which is abelian. Kernels and cokernels are computed in the obvious way; if $\sigma$ : $F \rightarrow G$ is a morphism, we have

$$
\begin{gathered}
{[\operatorname{ker}(\sigma)](B)=\operatorname{ker}(\sigma(B))=\{x \in F(B): \sigma(x)=0\},} \\
{[\operatorname{coker}(\sigma)](B)=\operatorname{coker}(\sigma(B))=G(B) / \sigma(F(B)) .}
\end{gathered}
$$

One sees that $\sigma$ is a monomorphism if and only if $\sigma(B)$ is injective for every $B$, and $\sigma$ is an epimorphism if and only if $\sigma(B)$ is surjective for every $B$.

Evidently, any model-valued $A$-functor may be viewed also as an $A$-functor. In some situations we shall want to consider $\phi: F \rightarrow G$ in which $F$ and $G$ are modulevalued $A$-functors but $\phi$ is a morphism of $A$-functors, not necessarily preserving the module structure. For clarity, we may say that $\phi$ is linear, if we wish to assume that it is a morphism of module-valued $A$-functors. In this section, all morphisms are linear.

If $M$ is an $A$-module, then $\underline{M}$ is a module-valued $A$-functor. If a given modulevalued $A$-functor $F$ is isomorphic to $\underline{M}$ for some finitely generated $A$-module $M$, we shall say that $F$ is strictly module-coherent.

Definition. A module-valued $A$-functor is module-coherent if it may be obtained as an iterated finite limit of strictly module-coherent $A$-functors. These limits are all taken in $\langle\langle$ module-valued $A$-functors $\rangle\rangle$.

We will show (2.9) that in fact this definition is equivalent to the (much simpler) definition of module-coherent given in the Introduction.

Problem 2.1. If a module-valued A-functor $F$ is coherent (when thought of simply as an A-functor), does it follow that $F$ is module-coherent?

We may define $\langle\langle$ module-coherent $A$-functors $\rangle\rangle$ : it is a full subcategory of $\langle\langle$ module-valued $A$-functors $\rangle\rangle$, which may be thought of as the finite completion of the subcategory $\langle\langle$ strictly module-coherent $A$-functors $\rangle\rangle$ of $\langle\langle$ module-valued $A$ functors $\rangle\rangle$.

Let $\mathcal{M C}_{A}^{0}$ denote the collection of strictly module-coherent $A$-functors. For each $n \geq 0$, let $\mathcal{M C}_{A}^{n+1}$ denote the collection of module-valued $A$-functors which may be obtained as finite limits (inside $\langle\langle$ module-valued $A$-functors $\rangle\rangle$ ) of objects in $\mathcal{M C}_{A}^{n}$. We have

$$
\mathcal{M C}_{A}^{0} \subset \mathcal{M C}_{A}^{1} \subset \mathcal{M C}_{A}^{2} \subset \cdots .
$$

Let $\mathcal{M C}_{A}=\bigcup_{n=0}^{\infty} \mathcal{M C}_{A}^{n}$. Then the objects in $\mathcal{M C}_{A}$ are exactly the module-coherent $A$-functors.

Definition. Let $F$ be a module-coherent $A$-functor. Then the level of $F$ is the smallest integer $n$ such that $F \in \mathcal{M C}_{A}^{n}$. 
To show that our definition of module-coherent is equivalent to the definition given in the Introduction, we will show (2.9) that the level of $F$ is always $\leq 1$. In the meantime, however, we will employ induction on the level of a given modulecoherent $A$-functor. For this we use the following analog of (1.1), whose proof is left to the reader.

Lemma 2.2. Let $F$ be a module-coherent A-functor of level $n \geq 1$. Then there exists a left exact sequence

$$
0 \rightarrow F \rightarrow G \rightarrow \underline{M}
$$

in which $G$ is a module-coherent A-functor of level $n-1$ and $M$ is a finitely generated A-module. Moreover, $F$ may be embedded as a sub-module-valued functor of a strictly module-coherent A-functor.

There is a functor $i_{A}$ from $\langle\langle$ finitely generated $A$-modules $\rangle\rangle$ to $\langle\langle$ module-coherent $A$-functors $\rangle$, given by $M \mapsto \underline{M}$. It is easily seen that $i_{A}$ is fully faithful, so we may view module-coherent $A$-functors as a sort of generalization of finitely generated $A$-modules. The functor $i_{A}$ is cocontinuous: it preserves colimits. However, $i_{A}$ does not carry monomorphisms to monomorphisms and is not continuous: it does not preserve limits. For example, if $J \subset A$ is an ideal, then $J$ is the kernel of the canonical map $A \rightarrow A / J$ of modules, but $\underline{J}$ is not the kernel of the induced map $\phi: \underline{A} \rightarrow A / J$. The kernel of $\phi$ is instead given by $B \mapsto J B$.

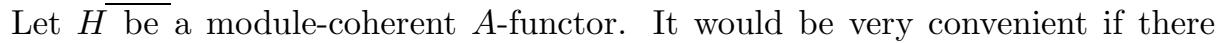
existed an epimorphism $\underline{A^{n}} \rightarrow H$ for some $n$. Unfortunately, this is not always the case. For example, it is not the case if $H(B)=\operatorname{Ann}_{B}(x)$, where $A=\mathbb{C}[x]$. (The module-coherence of this functor follows from example (7) of $\S 4$.) As a compromise, we are led to the following notion:

Definition. A module-valued $A$-functor $F$ is linearly representable if there exists a left exact sequence

$$
0 \rightarrow F \rightarrow \underline{A^{n}} \rightarrow \underline{A^{k}}
$$

in $\langle\langle$ module-valued $A$-functors $\rangle\rangle$, for some $n, k \geq 0$.

If $F$ is linearly representable, it is module-coherent, and it is representable by an $A$-algebra of the form

$$
A\left[x_{1}, \ldots, x_{n}\right] /\left(f_{1}, \ldots, f_{k}\right),
$$

where $f_{1}, \ldots, f_{k}$ are linear and homogeneous in $x_{1}, \ldots, x_{n}$. The following proposition is a basic tool, because it exhibits any module-coherent $A$-functor as a quotient of "something simple".

Proposition 2.3. Let $F$ be a module-coherent A-functor. Then there exist a linearly representable A-functor $R$ and an epimorphism $R \rightarrow F$.

There are some preliminaries.

Lemma 2.4. Let $F$ be a linearly representable A-functor, represented by $C=$ $A\left[x_{1}, \ldots, x_{n}\right] /\left(f_{1}, \ldots, f_{k}\right)$, where $f_{1}, \ldots, f_{k}$ are linear and homogeneous. Assume that the module structure on $F$ is the canonical one, induced from the embedding in $\underline{A^{n}}$ defined by $x_{1}, \ldots, x_{n}$. Let $C_{1}$ denote the degree 1 part of $C$. Let $N$ be an $A$-module. Then morphisms from $F$ to $\underline{N}$ are in bijective correspondence with elements of $N \otimes_{A} C_{1}$. 
Proof. We can think of $F$ and $\underline{N}$ as functors from the category of commutative $A$ algebras to $\langle\langle$ sets $\rangle\rangle$. If we take this point of view, then some morphisms (i.e. natural transformations) from $F$ to $\underline{N}$ define morphisms in $\langle\langle$ module-valued $A$-functors $\rangle$, and some do not. Those which do will be called linear, for purposes of this proof. The linear morphisms are those which preserve the module structure.

If we take this point of view, then morphisms from $F$ to $\underline{N}$ are in bijective correspondence with elements of $\underline{N}(C)=N \otimes_{A} C$, and it is clear that the elements of $N \otimes_{A} C_{1}$ define linear morphisms. To complete the proof, we must show that if an element $\eta \in N \otimes_{A} C$ corresponds to a linear morphism, then $\eta \in N \otimes_{A} C_{1}$.

The grading of $C$ induces a grading of $N \otimes_{A} C$. Let $\eta_{1} \in N \otimes_{A} C_{1}$ denote the degree 1 part of $\eta$. Let $\eta_{0}=\eta-\eta_{1}$. Then the degree 1 part of $\eta_{0}$ is 0 and $\eta_{0}$ defines a linear morphism $\psi: F \rightarrow \underline{N}$. We must show that $\psi=0$.

Let $B$ be a commutative $A$-algebra. Let $D=B[t]$. Then $\psi(d x)=d \psi(x)$ for all $d \in D$ and all $x \in F(D)$. In particular, $\psi(t x)=t \psi(x)$ for all $x \in F(B)$. We have $\psi(x) \in N \otimes_{A} B, \psi(t x) \in N \otimes_{A} D=\left(N \otimes_{A} B\right)[t]$. Since $\psi(t x)=t \psi(x), \psi(t x)$ is a homogeneous linear polynomial in $t$. The element $t x \in F(D)$ defines a ring homomorphism $\rho: C \rightarrow D$, which maps each generator $x_{i}$ of $C$ to a homogeneous linear polynomial in $t$. The map $\rho$ induces a map $N \otimes_{A} C \rightarrow N \otimes_{A} D$, which sends $\eta_{0}$ to $\psi(t x)$. But $\eta_{0}$ has no linear part, so it follows that $\psi(t x)$ has no linear part. Hence $\psi(t x)=0$. Hence $t \psi(x)=0$, so $\psi(x)=0$. Hence $\psi=0$.

Proposition 2.5. Let $P$ be an A-module. Suppose given a diagram

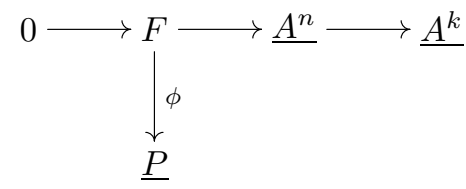

in $\langle\langle$ module-valued $A$-functors $\rangle\rangle$, with the row exact. Then there exists a morphism $h: \underline{A^{n}} \rightarrow \underline{P}$ which makes the diagram commute.

Proof. Let $C=A\left[x_{1}, \ldots, x_{n}\right]$ and

$$
\bar{C}=A\left[x_{1}, \ldots, x_{n}\right] /\left(f_{1}, \ldots, f_{k}\right),
$$

where $f_{1}, \ldots, f_{k}$ are homogeneous linear elements determined by the given map from $\underline{A^{n}}$ to $\underline{A^{k}}$. Then $F$ represents $\bar{C}$. According to (2.4), $\phi$ corresponds to an element of $P \otimes_{A} \bar{C}_{1}$. The canonical map $P \otimes_{A} C_{1} \rightarrow P \otimes_{A} \bar{C}_{1}$ is surjective, so $h$ exists.

Corollary 2.6. Let $\mathcal{D}$ be a finite diagram in $\langle\langle$ module-valued A-functors $\rangle$, in which the objects are linearly representable. Then the limit of $\mathcal{D}$ is linearly representable.

Proof. It is clear that a product of finitely many linearly representable $A$-functors is linearly representable. Since any linearly representable $A$-functor embeds in $\underline{A^{r}}$ for some $r$, we may reduce to showing that if $F$ is linearly representable and $\varphi: F \rightarrow \underline{A^{r}}$ is a morphism, then $\operatorname{ker}(\varphi)$ is linearly representable. Let

$$
0 \rightarrow F \rightarrow \underline{A^{n}} \stackrel{h}{\rightarrow} \underline{A^{k}}
$$

be as in the definition of linearly representable. By (2.5), $\varphi$ extends to a morphism $\psi: \underline{A^{n}} \rightarrow \underline{A^{r}}$. Hence $\operatorname{ker}(\varphi)=\operatorname{ker}(\psi) \cap \operatorname{ker}(h)$, so $\operatorname{ker}(\varphi)$ is linearly representable. 
Proof of (2.3). For purposes of the proof, let us say that a module-valued $A$-functor $G$ dominates a module-valued $A$-functor $F$ if there exists an epimorphism $G \rightarrow F$, and that a module-valued $A$-functor $F$ is linearly-affine-dominated if it is dominated by a linearly representable $A$-functor.

Let $n$ be the level of $F$. The case $n=0$ is clear-in that case $F$ is dominated by $\underline{A^{r}}$ for some $r$.

Suppose that $n \geq 1$. By (2.2), we may find a left exact sequence

$$
0 \rightarrow F \rightarrow G \rightarrow \underline{M}
$$

in which $G$ is a module-coherent $A$-functor of level $n-1$ and $M$ is a finitely generated $A$-module. By induction on $n$, we may assume that there exist a linearly representable $A$-functor $H$ and an epimorphism $H \rightarrow G$. Let $P$ be the fiber product of $H$ with $F$ over $G$. Then $P$ dominates $F$, so it suffices to show that $P$ is linearly-affine-dominated.

We have a left exact sequence

$$
0 \rightarrow P \rightarrow H \rightarrow \underline{M} .
$$

Choose an epimorphism $h: A^{r} \rightarrow M$. Let $L$ be the fiber product of $H$ with $\underline{A^{r}}$ over $\underline{M}$. Let $Q$ be the fiber product of $L$ with $P$ over $H$. We have a diagram with cartesian squares, in which some maps are labelled:

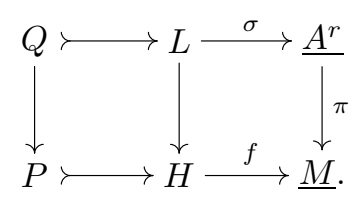

The bottom row (but not the top) is exact. The vertical arrows are all epimorphisms. Since $Q$ dominates $P$, it suffices to show that $Q$ is linearly-affine-dominated.

Let $K=\operatorname{ker}(\pi)$. Then $Q=\sigma^{-1}(K)$, so we have a cartesian diagram

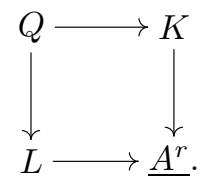

We will show that $K$ and $L$ are linearly-affine-dominated. It will follow (by taking suitable fiber products) that $Q$ is dominated by a fiber product of linearly representable $A$-functors. By (2.6), it will follow that $Q$ is linearly-affine-dominated.

There is a canonical epimorphism $\operatorname{ker}(h) \rightarrow K$. Choose an epimorphism $A^{s} \rightarrow$ $\operatorname{ker}(h)$. Then $K$ is dominated by $\underline{A}^{s}$. To complete the proof, we will show that $L$ is linearly-affine-dominated.

By (2.4), it follows that $f$ factors through $\pi$; let $g: H \rightarrow \underline{A^{r}}$ be such that $f=\pi \circ g$. Then

$$
\begin{aligned}
L(B) & =\left\{(x, y) \in H(B) \times \underline{A^{r}}(B): f(x)=\pi(y)\right\} \\
& =\left\{(x, y) \in H(B) \times \underline{A^{r}}(B): \pi(g(x)-y)=0\right\} .
\end{aligned}
$$

Hence the morphism of module-valued $A$-functors $H \times K \rightarrow L$ given by

$$
(x, y) \mapsto(x, g(x)+y)
$$

is an isomorphism. Since $K$ is linearly-affine-dominated, and $H$ is linearly representable, it follows that $L$ is linearly-affine-dominated. 
Theorem 2.7. Let $\varphi: F \rightarrow G$ be a morphism of module-coherent A-functors. Then $\operatorname{Coker}(\varphi)$ is module-coherent.

Proof. Let $n$ be the level of $G$. First suppose that $n=0$, so we may assume that $G=\underline{M}$ for some finitely generated $A$-module $M$. Choose an epimorphism $A^{m} \rightarrow M$ of $A$-modules and thus an epimorphism $\underline{A^{m}} \rightarrow \underline{M}$. Let $F^{\prime}$ be the fiber product of $F$ with $\underline{A^{m}}$ over $\underline{M}$. Let $C=\operatorname{Coker}(\varphi)$. Then we have a right exact sequence

$$
F^{\prime} \stackrel{f}{\rightarrow} \underline{A^{m}} \rightarrow C \rightarrow 0 .
$$

By (2.3), we may assume that $F^{\prime}$ is linearly representable. Choose a left exact sequence

$$
0 \rightarrow F^{\prime} \rightarrow \underline{A^{n}} \rightarrow \underline{A^{k}} .
$$

By (2.5), there exists a morphism $\underline{A^{n}} \rightarrow \underline{A^{m}}$ which makes the following diagram commute:

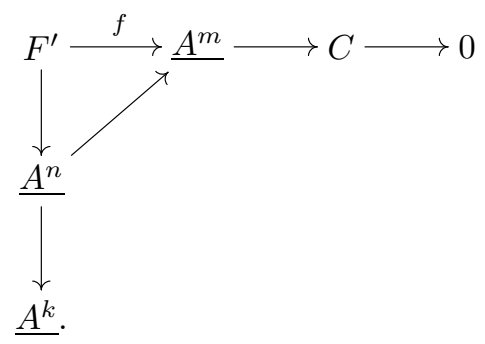

Let $D$ be the co-fiber product of $A^{m}$ and $A^{k}$ over $A^{n}$, computed in the category of $A$-modules. Then in fact $\underline{D}$ is the co-fiber product of $\underline{A^{m}}$ and $\underline{A^{k}}$ over $\underline{A^{n}}$. Let $g: \underline{A^{m}} \rightarrow \underline{D}$ be the canonical map. Then $\operatorname{ker}(g)=\operatorname{Im}(f)$, so $C$ is isomorphic to $\operatorname{Im}(g)$, which is module-coherent by example (4) in $\S 4$. This completes the case $n=0$.

Now suppose that $n \geq 1$. By (2.2), we may choose a left exact sequence

$$
0 \rightarrow G \rightarrow H \rightarrow \underline{M}
$$

in which $H$ is a module-coherent $A$-functor of level $n-1$ and $M$ is a finitely generated $A$-module. Abusing notation slightly, we have a left exact sequence

$$
0 \rightarrow G / F \rightarrow H / F \rightarrow \underline{M} .
$$

By induction on $n$, we may assume that $H / F$ is module-coherent. But then $\operatorname{Coker}(\varphi)=G / F$ is exhibited as the kernel of a morphism of module-coherent $A$-functors, so it too is module-coherent.

Corollary 2.8. If $\phi: F \rightarrow G$ is a morphism of module-coherent A-functors, then $\operatorname{Im}(\phi)$ is module-coherent.

Proof. Let $K=\operatorname{Ker}(\phi)$. Then $K$ is module-coherent. Hence Coker $[K \rightarrow F]$ is module-coherent by (2.7), but this equals $\operatorname{Im}(\phi)$.

The next result says that the definition of module-coherent given in this section coincides with the simpler definition given in the Introduction.

Corollary 2.9. Let $F$ be a module-coherent A-functor. Then $F$ has level $\leq 1$. 
Proof. By (2.2), we may embed $F$ as a subfunctor of $\underline{M}$, for some finitely generated $A$-module $M$. Let $Q=\underline{M} / F$. By (2.7), $Q$ is module-coherent. By (2.2), we may embed $Q$ as a subfunctor of $\underline{N}$, for some finitely generated $A$-module $N$. Hence $F$ is the kernel of a morphism from $\underline{M}$ to $\underline{N}$.

\section{QUASI-COHERENT FUNCTORS}

In this section we sketch a theory (parallel to the last two sections) of quasicoherent and module-quasi-coherent $A$-functors. The results of this section will be used in $\S 5$. In particular, it is the case that quasi-coherent $A$-functors (which are not coherent) are useful in the study of coherent $A$-functors. However, the reader interested only in the Picard group results may ignore this section and everything from (5.6) to the end of $\S 5$. The reason for this is explained in the paragraph preceding (5.6).

If a given $A$-functor is isomorphic to $\underline{M}$ for some $A$-module $M$, we shall say that $F$ is strictly quasi-coherent. Similarly, if a given module-valued $A$-functor $F$ is isomorphic to $\underline{M}$ for some $A$-module $M$, we shall say that $F$ is strictly modulequasi-coherent.

An $A$-functor is quasi-coherent if it may be obtained as an iterated finite limit of strictly coherent $A$-functors. These limits are all taken in $\langle\langle A$-functors $\rangle\rangle$. We shall not have much more to say about quasi-coherent $A$-functors per se in this paper.

A module-valued $A$-functor is module-quasi-coherent if it may be obtained as an iterated finite limit of strictly module-quasi-coherent $A$-functors. These limits are all taken in $\langle\langle$ module-valued $A$-functors $\rangle$.

The rest of this section is about module-quasi-coherent $A$-functors. All morphisms will be linear.

We may define the level of a module-quasi-coherent $A$-functor, as we have done for module-coherent $A$-functors. As we shall see (3.7), any module-quasi-coherent $A$-functor has level $\leq 1$. The analog of (2.2) for module-quasi-coherent $A$-functors is:

Lemma 3.1. Let $F$ be a module-quasi-coherent A-functor of level $n \geq 1$. Then there exists a left exact sequence

$$
0 \rightarrow F \rightarrow G \rightarrow \underline{M}
$$

in which $G$ is a module-quasi-coherent A-functor of level $n-1$ and $M$ is an Amodule. Moreover, F may be embedded as a sub-module-valued functor of a strictly module-quasi-coherent A-functor.

We make the following convention: if $N$ is a set, then $A^{N}$ denotes a direct sum of copies of $A$, one for each element of $N$. If $S$ is a subset of $N$, then we may view $A^{S}$ as a submodule of $A^{N}$, and thence we may view $\underline{A^{S}}$ as a subfunctor of $\underline{A^{N}}$.

Proposition 3.2. Let $P$ be an A-module. Let $N$ and $K$ be sets. Suppose given a diagram

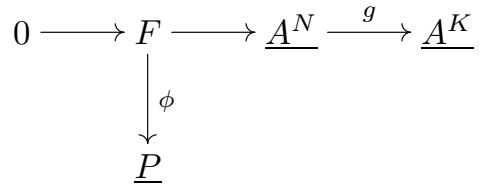


in $\langle\langle$ module-valued $A$-functors $\rangle\rangle$, with the row exact. Then there exists a morphism $h: \underline{A^{N}} \rightarrow \underline{P}$ which makes the diagram commute.

Proof. Let $\operatorname{fin}(N)$ denote the collection of finite subsets of $N$. Then

$$
\left\{\underline{A^{S}}\right\}_{S \in \operatorname{fin}(N)}
$$

forms a directed system of subfunctors of $\underline{A^{N}}$, whose union is $\underline{A^{N}}$. Let $F_{S}=F \cap \underline{A^{S}}$ for each $S$. Then $\left\{F_{S}\right\}_{S \in \operatorname{fin}(N)}$ forms a directed system of subfunctors of $F$, whose union is $F$.

It is clear that $\left.g\right|_{A^{S}}$ factors through the subfunctor $\underline{A}^{S^{*}}$ of $\underline{A^{K}}$, for some finite subset $S^{*}$ of $K$. It follows that $F_{S}$ is linearly representable by a ring $\overline{C_{S}}=$ $A\left[\left\{x_{s}\right\}_{s \in S}\right] / I_{S}$, where $I_{S}$ is generated by linear homogeneous elements. Let $C_{S}$ be the polynomial ring $A\left[\left\{x_{s}\right\}_{s \in S}\right]$.

By $(2.4),\left.\phi\right|_{F_{S}}$ corresponds to an element of $P \otimes_{A}\left(\overline{C_{S}}\right)_{1}$. By lifting this element to an element of $P \otimes_{A}\left(C_{S}\right)_{1}$, we see that $\left.\phi\right|_{F_{S}}$ can be extended to a morphism $h_{S}: \underline{A^{S}} \rightarrow \underline{P}$. As $S$ varies, we have to choose these extensions $h_{S}$ so that they are compatible with each other. To do this is equivalent to showing that the canonical map

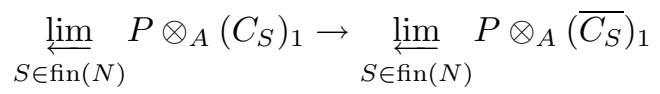

is surjective. In general, it is not true that an inverse limit of surjective module maps is surjective, but ([4], 10.2) it is the case if the transition maps in the system of kernels are surjective. To show this, it suffices to show that if $S, S^{\prime} \in \operatorname{fin}(N)$, with $S \subset S^{\prime}$, then the canonical map $P \otimes_{A}\left(I_{S^{\prime}}\right)_{1} \rightarrow P \otimes_{A}\left(I_{S}\right)_{1}$ is surjective. This follows from the fact that the canonical map $I_{S^{\prime}} \rightarrow I_{S}$ is surjective.

Definition. A module-valued $A$-functor $F$ is linearly quasi-representable if there exist sets $N$ and $K$ and a left exact sequence

$$
0 \rightarrow F \rightarrow \underline{A^{N}} \rightarrow \underline{A^{K}}
$$

in $\langle\langle$ module-valued $A$-functors $\rangle\rangle$.

Using (3.2), one may prove the following analog of (2.6):

Corollary 3.3. Let $\mathcal{D}$ be a finite diagram in $\langle\langle$ module-valued A-functors $\rangle$, in which the objects are linearly quasi-representable. Then the limit of $\mathcal{D}$ is linearly quasi-representable.

Evidently, any linear quasi-representable $A$-functor is module-quasi-coherent.

Proposition 3.4. Let $F$ be a module-quasi-coherent A-functor. Then there exist a linearly quasi-representable A-functor $R$ and an epimorphism $R \rightarrow F$.

Proof (Sketch). Take the proof of (2.3), and modify it in the following ways. In the various places where $A^{r}$ is written, one has to allow $r$ to be an arbitrary set. Do the same with $A^{s}$. Change each reference to module-coherent to module-quasi-coherent. Drop the assumption that $M$ is finitely generated. The construction of $g$ requires the use of (3.2). Use (3.3) instead of (2.6). Use (3.1) instead of (2.2).

Proposition 3.5. Let $\varphi: F \rightarrow G$ be a morphism of module-quasi-coherent Afunctors. Then $\operatorname{Coker}(\varphi)$ is module-quasi-coherent. 
Proof (Sketch). Take the proof of (2.7), and modify it in the following ways. Change each reference to module-coherent to module-quasi-coherent. Drop the assumption that $M$ is finitely generated. In the notations $A^{m}, A^{n}$, and $A^{k}$, one must allow $m, n$, and $k$ to be arbitrary sets. Use (3.2) instead of (2.5). Use (3.4) instead of (2.3). Use (3.1) instead of (2.2). Modify reference to example (4) from $§ 4$ appropriately.

Corollary 3.6. Let $\phi: F \rightarrow G$ be a morphism of module-quasi-coherent A-functors. Then $\operatorname{Im}(\phi)$ is module-quasi-coherent.

Corollary 3.7. Let $F$ be a module-quasi-coherent A-functor. Then $F$ has level $\leq 1$

Lemma 3.8. Let $F$ be a module-coherent A-functor. Let $\left\{F_{\lambda}\right\}_{\lambda \in \Lambda}$ be a system of subfunctors of $F$, whose union is $F$. Then $F=F_{\lambda_{0}}$, for some $\lambda_{0} \in \Lambda$.

Proof. By (2.3), we may find a linearly representable $A$-functor $H$, and an epimorphism $\pi: H \rightarrow F$. Let $H_{\lambda}=\pi^{-1}\left(F_{\lambda}\right)$, for each $\lambda \in \Lambda$. (That is, $H_{\lambda}$ is the fiber product of $F_{\lambda}$ with $H$ over $F$.) Then $H$ is the union of the $H_{\lambda}$. Identify $H$ with the functor representing some $A$-algebra $C: H(B)=\operatorname{Hom}_{A \text {-alg }}(C, B)$. Choose $\lambda_{0} \in \Lambda$ such that $1_{C} \in H_{\lambda_{0}}(C)$. It follows that there exists a natural transformation of functors $s: H \rightarrow H_{\lambda_{0}}$ (possibly not preserving module structures) such that $i \circ s=1_{H}$, where $i: H_{\lambda_{0}} \rightarrow H$ is the inclusion. Hence $i$ is an isomorphism. Hence $H_{\lambda_{0}}=H$. Since $\pi$ is an epimorphism, it follows that $F_{\lambda_{0}}=F$.

Remark 3.9. If $F$ is module-coherent and $G$ is module-quasi-coherent, and $\varphi: F \rightarrow$ $G$ is a morphism, then $\operatorname{ker}(\varphi)$ need not be module-coherent. For an example, let $A=\mathbb{Z}, F=\underline{\mathbb{Z}}, G=\underline{\mathbb{Q}}$, and let $\varphi$ be the map given by $n \mapsto n$. Indeed,

$$
\operatorname{ker}(\varphi)(B)=\{b \in B: n b=0 \text { for some } n \in \mathbb{N}\} .
$$

Then $\operatorname{ker}(\varphi)$ is the direct limit of its subfunctors $F_{n}=\{b \in B: n b=0\}$, for $n \geq 1$, and since $\operatorname{ker}(\varphi) \neq F_{n}$ for all $n$, it follows from $(3.8)$ that $\operatorname{ker}(\varphi)$ is not module-coherent.

\section{EXAMPLES}

First we give some examples, all of which are easily seen to satisfy the simple definition of module-coherent which we gave in the Introduction.

(1) $B \mapsto M \otimes_{A} B$, where $M$ is a finitely generated $A$-module.

(2) $B \mapsto \operatorname{Ker}\left(f \otimes_{A} B\right)$, where $f: M \rightarrow N$ is a homomorphism of finitely generated $A$-modules. [We shall denote this $A$-functor by $\underline{\operatorname{Ker}}(f)$.]

(3) $B \mapsto I B$, where $I$ is an ideal of $A$. [Consider the kernel of the map $\underline{A} \rightarrow A / I$.]

(4) $B \mapsto \operatorname{Im}\left(f \otimes_{A} B\right)$, where $f: M \rightarrow N$ is a homomorphism of finitely generated $A$-modules. [We shall denote this functor by $\underline{\operatorname{Im}}(f)$. Let $g: N \rightarrow N / \operatorname{Im}(f)$ be the canonical map. Then $\underline{\operatorname{Im}}(f)=\underline{\operatorname{Ker}}(g)$, so $\underline{\operatorname{Im}}(f)$ is module-coherent.]

(5) $B \mapsto \operatorname{Hom}_{B-\bmod }\left(M \otimes_{A} B, N \otimes_{A} B\right)$, where $M$ and $N$ are finitely generated $A$-modules. [We shall denote this functor by $\underline{\operatorname{Hom}}(M, N)$. To see why it is module-coherent, choose a presentation $A^{k} \rightarrow A^{n} \rightarrow M \rightarrow 0$ for $M$. Consider the induced map $f: \operatorname{Hom}\left(A^{n}, N\right) \rightarrow \operatorname{Hom}\left(A^{k}, N\right)$. Then $\underline{\operatorname{Hom}}(M, N)$ is isomorphic to $\underline{\operatorname{Ker}}(f)$.]

(6) $B \mapsto \operatorname{End}_{B \text { - mod }}\left(M \otimes_{A} B\right)$, where $M$ is a finitely generated $A$-module. [We shall denote this functor by $\underline{\operatorname{End}}(M)$.] 
(7) $B \mapsto \operatorname{Ann}_{B}(I)$, where $I \subset A$ is a fixed ideal. [We shall denote this functor by $\underline{\text { Ann }}(I)$. The point is that $\operatorname{Ann}_{B}(I)=\operatorname{Hom}_{B-\bmod }(B / I, B)$; use example (5).]

Now we give some simple examples of coherent $A$-functors.

(8) $B \mapsto \operatorname{Hom}_{A \text {-alg }}(C, B)$, where $C$ is a commutative $A$-algebra of finite type. [Choose a presentation $C=A\left[x_{1}, \ldots, x_{n}\right] /\left(f_{1}, \ldots, f_{k}\right)$. Then $f_{1}, \ldots, f_{k}$ define a morphism of $A$-functors $A^{n} \rightarrow \underline{A^{k}}$, whose kernel is the given functor.]

(9) $B \mapsto\left\{x \in B: x^{2} \in I B\right\}$. [Consider the kernel of the map $\underline{A} \rightarrow A / I$ given by $x \mapsto x^{2}$.]

(10) $B \mapsto\left\{a \in A^{n}: f(a)=0\right\}$, where $f \in M\left[x_{1}, \ldots, x_{n}\right]$. [Consider kernels of maps $\underline{A^{n}} \rightarrow \underline{M}$; this generalizes the preceding example.]

The coherence of the remaining examples of this section follows without great difficulty from the tools developed so far. However, the remaining examples seem to be deeper, in the sense that their coherence cannot be deduced directly from the definitions.

Presumably, all of the usual linear algebra operations $\left(\mathrm{Hom}, \otimes, \Lambda^{n}, \ldots\right)$ have analogs for module-valued $A$-functors. A thorough study (which we do not give) would include definitions of these operations and an analysis of which preserve module-coherence. We restrict our attention to some special cases.

Let $F$ and $G$ be module-valued $A$-functors. Then there is a module-valued $A$ functor $F \otimes G$, given by $B \mapsto F(B) \otimes_{B} G(B)$. If $F$ and $G$ are module-coherent, one can ask if $F \otimes G$ is module-coherent. It turns out (4.4) that this is not the case. However, there is the following special case:

Proposition 4.1. Let $F$ be a module-coherent $A$-functor, and let $M$ be a finitely generated A-module. Then $F \otimes \underline{M}$ is module-coherent.

Proof. Choose a right exact sequence

$$
A^{k} \rightarrow A^{n} \rightarrow M \rightarrow 0
$$

of $A$-modules. We obtain a right exact sequence

$$
F \otimes \underline{A^{k}} \rightarrow F \otimes \underline{A^{n}} \rightarrow F \otimes \underline{M} \rightarrow 0
$$

of module-valued $A$-functors, and thence a right exact sequence

$$
F^{k} \rightarrow F^{n} \rightarrow F \otimes \underline{M} \rightarrow 0 .
$$

Since $F^{k}$ and $F^{n}$ are module-coherent, it follows by (2.7) that $F \otimes \underline{M}$ is modulecoherent.

To show that tensor products do not (in general) preserve module-coherence, we need the following lemma, which will also be used in a counterexample presented in an Appendix.

Lemma 4.2. Let $A$ be a noetherian local ring of dimension $d$, having maximal ideal $\mathfrak{m}$. Let $F$ be a module-coherent A-functor. Then there exists a constant $c$ such that, for each $n \in \mathbb{N}$ and every ideal I of $A$ with $\mathfrak{m}^{n} \subset I$, we have $\mu[F(A / I)] \leq c n^{d}$, where $\mu$ gives the minimal number of generators of an A-module.

Remark 4.3. Perhaps the bound $c n^{d}$ can be replaced by $c n^{d-1}$. 
Proof of Lemma 4.2. We may assume that $F=\underline{\operatorname{Ker}}(f)$, for some homomorphism $f: M \rightarrow N$ of finitely generated $A$-modules. Let $\lambda$ denote length. Choose a surjection $A^{s} \rightarrow M$. Since we have

$$
\mathfrak{m}^{n+1} M \subset \mathfrak{m} f^{-1}(I N) \subset f^{-1}(I N) \subset M,
$$

it follows that

$$
\begin{aligned}
& \mu\left[\operatorname{Ker}\left(f \otimes_{A} A / I\right)\right]=\mu\left[f^{-1}(I N) / I M\right] \leq \mu\left[f^{-1}(I N)\right] \\
& \quad=\mu\left[f^{-1}(I N) / \mathfrak{m} f^{-1}(I N)\right]=\lambda\left[f^{-1}(I N) / \mathfrak{m} f^{-1}(I N)\right] \\
& \quad \leq \lambda\left(M / \mathfrak{m}^{n+1} M\right) \leq \lambda\left(A^{s} / \mathfrak{m}^{n+1} A^{s}\right)=s \lambda\left(A / \mathfrak{m}^{n+1}\right) .
\end{aligned}
$$

The lemma follows from the theory of the Hilbert-Samuel polynomial.

Now we show that the tensor product of two module-coherent $A$-functors need not be module-coherent:

Proposition 4.4. Let $A=\mathbb{C}[[s, t, u]]$, and let $I$ be the ideal $(s)$ of $A$. Then the module-valued A-functor $\underline{\operatorname{Ann}}(I) \otimes \underline{\operatorname{Ann}}(I)$ is not module-coherent.

Proof. Let $F$ denote the given functor. Fix $n \in \mathbb{N}$, and let $B=A /(s, t, u)^{n}$. Then a minimal generating set for $\operatorname{Ann}_{B}(s)$ is

$$
\left\{s^{i} t^{j} u^{k}\right\}_{0 \leq i, j, k \leq n-1, i+j+k=n-1},
$$

which has cardinality $n(n+1) / 2$. Then

$$
\mu[F(B)]=\mu\left[\operatorname{Ann}_{B}(s)\right]^{2}=[n(n+1) / 2]^{2} .
$$

By (4.2), $F$ is not module-coherent.

Let $M$ and $N$ be finitely generated $A$-modules. For $n \geq 0$, one can ask if the functor $\operatorname{Tor}_{n}(M, N)$ given by $B \mapsto \operatorname{Tor}_{n}^{B}\left(M \otimes_{A} B, N \otimes_{A} B\right)$ is module-coherent. This seems unlikely for $n \geq 2$ (but we do not have a counterexample). For $n=0$, (4.1) applies. For $n=1$, we have

Proposition 4.5. Let $M$ and $N$ be finitely generated $A$-modules. Then $\underline{\operatorname{Tor}}_{1}(M, N)$ is module-coherent.

Proof. Choose an epimorphism $A^{n} \rightarrow M$ and thence a short exact sequence

$$
0 \rightarrow K \rightarrow \underline{A^{n}} \rightarrow \underline{M} \rightarrow 0
$$

in which $K$ is module-coherent. One obtains a left exact sequence

$$
0 \rightarrow \underline{\operatorname{Tor}}_{1}(M, N) \rightarrow K \otimes \underline{N} \rightarrow \underline{A^{n}} \otimes \underline{N} .
$$

The proposition now follows from (4.1).

If $F$ and $G$ are module-valued $A$-functors, we let $\operatorname{Hom}(F, G)$ denote the modulevalued $A$-functor given by

$$
B \mapsto \operatorname{Hom}_{\text {module-valued } B \text {-functors }}(F, G),
$$

where $F$ and $G$ may be viewed as module-valued $B$-functors because any $B$-algebra is an $A$-algebra. In a natural way, $\operatorname{Hom}(F, G)$ is itself a module-valued $A$-functor. We let $\operatorname{End}(F)$ denote $\operatorname{Hom}(F, F)$.

Example 4.6. Let $M$ and $N$ be finitely generated $A$-modules. Then there is a canonical isomorphism of module-valued $A$-functors

$$
\underline{\operatorname{Hom}}(M, N) \rightarrow \operatorname{Hom}(\underline{M}, \underline{N}) .
$$


Proposition 4.7. Let $F$ and $G$ be module-coherent A-functors. Then $\operatorname{Hom}(F, G)$ is module-coherent.

Proof. First observe that the bifunctor

Hom : $\langle\langle\text { module-valued } A \text {-functors }\rangle\rangle^{\circ} \times\langle\langle$ module-valued $A$-functors $\rangle\rangle$

$$
\rightarrow\langle\langle\text { module-valued } A \text {-functors }\rangle\rangle
$$

is left exact in both variables.

By (2.9), there is a left exact sequence

$$
0 \rightarrow G \rightarrow \underline{M}_{1} \rightarrow \underline{M}_{2}
$$

for some finitely generated $A$-modules $M_{1}$ and $M_{2}$. This yields a left exact sequence

$$
0 \rightarrow \operatorname{Hom}(F, G) \rightarrow \operatorname{Hom}\left(F, \underline{M}_{1}\right) \rightarrow \operatorname{Hom}\left(F, \underline{M}_{2}\right) .
$$

Thus it suffices to show that $\operatorname{Hom}\left(F, \underline{M}_{i}\right)$ is module-coherent for each $i$. Let $M=M_{i}$.

It follows from (2.3) that there exists a right exact sequence

$$
L_{2} \rightarrow L_{1} \rightarrow F \rightarrow 0
$$

in which $L_{1}$ and $L_{2}$ are linearly representable. We obtain a left exact sequence

$$
0 \rightarrow \operatorname{Hom}(F, \underline{M}) \rightarrow \operatorname{Hom}\left(L_{1}, \underline{M}\right) \rightarrow \operatorname{Hom}\left(L_{2}, \underline{M}\right) .
$$

Therefore we may reduce to proving that $\operatorname{Hom}(L, \underline{M})$ is module-coherent for any linearly representable $A$-functor $L$. The $A$-functor $L$ is representable by a ring

$$
C=A\left[x_{1}, \ldots, x_{n}\right] /\left(f_{1}, \ldots, f_{k}\right),
$$

where $f_{1}, \ldots, f_{k}$ are homogeneous linear polynomials in $x_{1}, \ldots, x_{n}$. From (2.4), it follows that

$$
\operatorname{Hom}(L, \underline{M}) \cong \underline{M \otimes_{A} C_{1}},
$$

so $\operatorname{Hom}(L, \underline{M})$ is module-coherent.

Corollary 4.8. For any module-coherent A-functor $F, \operatorname{End}(F)$ is module-coherent.

If $F$ is a module-valued $A$-functor, we let $\operatorname{Aut}(F)$ denote the $A$-functor given by

$$
B \mapsto \text { Aut module-valued } B \text {-functors }(F),
$$

where $F$ is viewed as a $B$-functor. Then $\operatorname{Aut}(F)$ is an $A$-functor.

Corollary 4.9. For any module-coherent A-functor $F, \operatorname{Aut}(F)$ is coherent.

Proof. Take the kernel of the map

$$
\operatorname{End}(F) \times \operatorname{End}(F) \rightarrow \operatorname{End}(F) \times \operatorname{End}(F)
$$

given by $(\alpha, \beta) \mapsto(\alpha \circ \beta-\mathrm{id}, \beta \circ \alpha-\mathrm{id})$.

By an algebra-valued $A$-functor $F$, we shall mean a module-valued $A$-functor $F$ which has the additional structure of a $B$-algebra on $F(B)$, for each $B$. We do not assume that these algebras $F(B)$ are commutative.

If $F, G$, and $H$ are module-valued $A$-functors, then $\operatorname{Bil}(F \times G, H)$ will denote the functor of bilinear maps from $F \times G$ to $H$, which sends $B$ to the $B$-module consisting of all morphisms of $B$-functors from $F \times G$ to $H$ which are bilinear. Then $\operatorname{Bil}(F \times G, H)$ is a module-valued $A$-functor. 
Corollary 4.10. Suppose that $F, G$, and $H$ are module-coherent $A$-functors. Then $\operatorname{Bil}(F \times G, H)$ is module-coherent.

Proof. We may identify $\operatorname{Bil}(F \times G, H)$ with $\operatorname{Hom}(F, \operatorname{Hom}(G, H))$.

If $F$ and $G$ are algebra-valued $A$-functors, we let $\mathbf{H o m}_{\text {alg }}(F, G)$ denote the $A$ functor given by

$$
B \mapsto \operatorname{Hom}_{\text {algebra-valued } B \text {-functors }}(F, G),
$$

where $F$ and $G$ are viewed as algebra-valued $B$-functors. Similarly, we may define Aut $_{\text {alg }}(F)$.

Corollary 4.11. Let $R$ and $S$ be module-coherent, algebra-valued $A$-functors. Then $\mathbf{H o m}_{\text {alg }}(R, S)$ is coherent.

Proof. Let $\mu_{R}: R \times R \rightarrow R$ and $\mu_{S}: S \times S \rightarrow S$ denote the multiplication maps. Then $\mathbf{H o m}_{\text {alg }}(R, S)$ is the kernel of the map

$$
\operatorname{Hom}(R, S) \rightarrow \operatorname{Bil}(R \times R, S)
$$

given by $f \mapsto\left[f \circ \mu_{R}\right]-\left[\mu_{S} \circ(f \times f)\right]$.

Example 4.12. Let $R$ and $S$ be module-finite $A$-algebras (not necessarily commutative). Then the $A$-functor defined by

$$
B \mapsto \operatorname{Hom}_{B-\operatorname{alg}}\left(R \otimes_{A} B, S \otimes_{A} B\right)
$$

is coherent.

Corollary 4.13. For any module-coherent, algebra-valued A-functor $R, \mathbf{A u t}_{\text {alg }}(R)$ is coherent.

Example 4.14. Let $R$ be a module-finite $A$-algebra (not necessarily commutative). Then the $A$-functor given by $B \mapsto \operatorname{Aut}_{B \text { - alg }}\left(R \otimes_{A} B\right)$ is coherent.

\section{THE GLOBAL CASE}

We have defined the notion of $X$-functor in the Introduction; these form a category $\langle\langle X$-functors $\rangle\rangle$. If $X=\operatorname{Spec}(A)$, then there is a canonical equivalence of categories between $\langle\langle A$-functors which are Zariski sheaves $\rangle\rangle$ and $\langle\langle X$-functors which are Zariski sheaves $\rangle\rangle$; we can pass back and forth freely between these two categories. Similarly, for an arbitrary noetherian scheme $X$, we may (instead of looking at $X$-functors which are Zariski sheaves) look at functors which are Zariski sheaves and whose source is

$$
\langle\langle X \text {-schemes which are quasi-compact }\rangle\rangle^{\circ} \text {. }
$$

Let $\phi: X \rightarrow Y$ be a morphism of noetherian schemes. We consider pull-back and push-forward of functors:

- Let $F$ be a $Y$-functor. Then there is an $X$-functor $\phi^{*} F$, given by $\left(\phi^{*} F\right)(T)=$ $F(T)$ for all $X$-schemes $T$. Sometimes we will write $\left.\phi\right|_{X}$ instead of $\phi^{*} F$, and refer to the restriction of $F$ to $X$.

- Let $G$ be an $X$-functor. Then there is a $Y$-functor $\phi_{*} G$, given by $\left(\phi_{*} G\right)(S)=$ $F\left(X \times_{Y} S\right)$, for all $Y$-schemes $S$.

- Let $F$ be a $Y$-functor. Then the $Y$-functor $\phi_{*} \phi^{*}(F)$ is given by $S \mapsto$ $F\left(X \times_{Y} S\right)$, for all $Y$-schemes $S$. Instead of writing $\phi_{*} \phi^{*}(F)$, we may refer to " $\left.F\right|_{X}$, viewed as a $Y$-functor." 
Note that both $\phi^{*}$ and $\phi_{*}$ are exact functors.

If $Y$ is an $X$-scheme, $\pi: Y \rightarrow X$ is a morphism of schemes, and $\mathcal{M}$ is a quasi-coherent $\mathcal{O}_{X}$-module, we let $\mathcal{M}_{Y}$ denote $\pi^{*} \mathcal{M}$. For any such $\mathcal{M}$, there is an $X$-functor $\underline{\mathcal{M}}$ given by $\underline{\mathcal{M}}(Y)=\Gamma\left(Y, \mathcal{M}_{Y}\right)$.

An $X$-functor is strictly coherent if it is isomorphic to $\underline{\mathcal{M}}$ for some coherent $\mathcal{O}_{X}$-module $\mathcal{M}$. An $X$-functor is coherent if it is an iterated finite limit of strictly coherent $X$-functors, where the limits are taken in $\langle\langle X$-functors $\rangle\rangle$. Similarly, one may define quasi-coherent $X$-functors, by allowing $\mathcal{M}$ to be quasi-coherent.

We may define the level of a coherent $X$-functor, exactly as we have done for coherent $A$-functors. We may also define the level of a quasi-coherent $X$-functor, and it is distantly conceivable that there exists a coherent $X$-functor whose level is lower when viewed as a quasi-coherent $X$-functor.

It is very important to note that if $X=\operatorname{Spec}(A)$, then coherent $X$-functors are essentially the same as coherent $A$-functors. This follows from the fact that coherent $X$-functors are sheaves for the Zariski topology. Indeed we have:

Proposition 5.1. Let $F$ be a quasi-coherent $X$-functor. Then $F$ is a sheaf for the fpqc topology.

Proof. If $F=\underline{\mathcal{M}}$, for some quasi-coherent $\mathcal{O}_{X}$-module $\mathcal{M}$, then the statement is true. The proposition follows because any limit of sheaves is a sheaf.

Definition. A module-valued $X$-functor is an (abelian group)-valued $X$-functor $F$, together with the structure of a $\Gamma\left(Y, \mathcal{O}_{Y}\right)$-module on each set $F(Y)$, with the property that for each map of $X$-schemes $Y_{1} \rightarrow Y_{2}$, the induced map $F\left(Y_{2}\right) \rightarrow F\left(Y_{1}\right)$ is a homomorphism of $\Gamma\left(Y_{2}, \mathcal{O}_{Y_{2}}\right)$-modules.

The module-valued $X$-functors form an abelian category. When we have $X=$ $\operatorname{Spec}(A)$, there is a canonical equivalence of categories between

$\langle\langle$ module-valued $A$-functors which are Zariski sheaves $\rangle\rangle$

and

$$
\langle\langle\text { module-valued } X \text {-functors which are Zariski sheaves }\rangle \text {. }
$$

If $\mathcal{M}$ is a quasi-coherent $\mathcal{O}_{X}$-module, we let $\underline{\mathcal{M}}$ denote the module-valued $X$ functor given by $Y \mapsto \Gamma\left(Y, \mathcal{M}_{Y}\right)$. A module-valued $X$-functor $F$ is strictly modulecoherent if there exists a coherent $\mathcal{O}_{X}$-module $\mathcal{M}$ such that $F \cong \underline{\mathcal{M}}$.

Definition. A module-valued $X$-functor is module-coherent if it may be obtained as an iterated finite limit of strictly module-coherent $X$-functors. These limits are all taken in $\langle\langle$ module-valued $X$-functors $\rangle\rangle$.

In a similar way, one may define module-quasi-coherent $X$-functors. If $X=$ $\operatorname{Spec}(A)$, then module-coherent $X$-functors are essentially the same as modulecoherent $A$-functors. For arbitrary $X$, the theory of module-coherent $X$-functors runs parallel to the theory of module-coherent $A$-functors, but there is one difference. When one takes the cokernel of a morphism of module-coherent $X$-functors, it is necessary to take the associated sheaf (with respect to the Zariski topology), in order to obtain a module-coherent $X$-functor.

The level of a module-coherent (or module-quasi-coherent) $X$-functor is defined analogously to the definition of level for a module-coherent $A$-functor. As in that case, we will find ultimately that the level is always $\leq 1$. 
An $X$-functor $F$ is locally coherent if $F$ is a Zariski sheaf and there exists an open cover $U_{1}, \ldots, U_{n}$ of $X$ such that $\left.F\right|_{U_{i}}$ is a coherent $U_{i}$-functor for each $i$. Similarly, a module-valued $X$-functor $F$ is locally module-coherent if $F$ is a Zariski sheaf and if there exists an open cover $U_{1}, \ldots, U_{n}$ of $X$ such that $\left.F\right|_{U_{i}}$ is a module-coherent $U_{i}$-functor for each $i$. We will show shortly that any locally module-coherent $X$ functor is module-coherent, assuming that $X$ is separated. (One can also define locally quasi-coherent and locally module-quasi-coherent $X$-functors.) We do not know the answer to the analogous question for locally coherent $X$-functors.

Conjecture 5.2. Every locally coherent $X$-functor is coherent.

If the conjecture were true, it would follow immediately that if an $X$-functor $F$ represents an affine $X$-scheme of finite type, then $F$ is coherent. (This is true if $X$ is affine: see example ( 8 ) from $\S 4$.)

Problem 5.3. Let $\phi: Y \rightarrow X$ be a faithfully flat morphism of noetherian schemes. Let $F$ be an $X$-functor which is a sheaf for the fpqc topology. Assume that $\phi^{*} F$ is a coherent $Y$-functor. Does it follow that $F$ is a coherent $X$-functor?

We now consider push-forward and pull-back of coherent and quasi-coherent $X$-functors. These operations also make sense for module-valued $X$-functors.

Proposition 5.4. Let $\phi: X \rightarrow Y$ be a morphism of noetherian schemes. Let $F$ be a coherent $Y$-functor. Then $\phi^{*} F$ is coherent. Similarly, if $F$ is a module-coherent $Y$-functor, then $\phi^{*} F$ is module-coherent.

Proof. Suppose that $F$ is a coherent $Y$-functor. (The parallel case for modulecoherent $Y$-functors is left to the reader.) Let $n$ be the level of $F$. First suppose that $n=0$, so $F \cong \underline{\mathcal{M}}$ for some coherent $\mathcal{O}_{Y}$-module $\mathcal{M}$. But then $\phi^{*} F \cong \phi^{*} \mathcal{M}$, so $\phi^{*} F$ is coherent. Now suppose that $n \geq 1$. By an unstated analog of (1.1), there is a left exact sequence

$$
F \longmapsto G \rightarrow \underline{\mathcal{M}}
$$

of $X$-functors in which $G$ is coherent of level $n-1$ and $\mathcal{M}$ is a coherent $\mathcal{O}_{X^{-}}$ module. By induction on $n$, we may assume that $\phi^{*} G$ is coherent. Since $\phi^{*}$ is an exact functor, we have a left exact sequence:

$$
\phi^{*} F \longmapsto \phi^{*} G \rightarrow \phi^{*} \underline{\mathcal{M}} .
$$

Hence $\phi^{*} F$ is coherent.

Proposition 5.5. Let $\phi: X \rightarrow Y$ be a morphism of noetherian schemes. Let $F$ be an $X$-functor.

(a) If $F$ is quasi-coherent and $\phi$ is affine, then $\phi_{*} F$ is quasi-coherent.

(b) If $F$ is coherent and $\phi$ is finite, then $\phi_{*} F$ is coherent.

(c) Parallel statements apply if $F$ is a module-valued $X$-functor.

Proof. (a) Let $n$ be the level of $F$. If $n=0, F=\underline{\mathcal{M}}$ for some quasi-coherent $\mathcal{O}_{X}$-module $\mathcal{M}$, so $\left(\phi_{*} F\right)(T)=\Gamma\left(X \times_{Y} T, \mathcal{M}_{X \times_{Y} T}\right)$ for any $Y$-scheme $T$. By ([19], I:9.1.1), it follows that $\left(\phi_{*} F\right)(T) \cong \Gamma\left(T,\left(\phi_{*} \mathcal{M}\right)_{T}\right)$. Hence $\phi_{*} F$ is coherent.

Now suppose that $n \geq 1$. By the (unstated) analog of (1.1) for quasi-coherent $X$-functors, there is a left exact sequence

$$
F \longmapsto G \rightarrow \underline{\mathcal{M}}
$$


in which $G$ is a quasi-coherent $X$-functor of level $n-1$ and $\mathcal{M}$ is a quasi-coherent $\mathcal{O}_{X}$-module. Then we have a left exact sequence

$$
\phi_{*} F \longmapsto \phi_{*} G \rightarrow \phi_{*} \underline{\mathcal{M}} .
$$

By induction on $n$, we may assume that $\phi_{*} G$ is quasi-coherent. By the $n=0$ case, we may identify $\phi_{*} \underline{\mathcal{M}}$ with $\phi_{*} \mathcal{M}$. Hence $\phi_{*} F$ is quasi-coherent.

Parts (b) and (c) are left to the reader.

The next result is key, since it permits us to reduce to the affine case, and thereby obtain the analogs of the results for module-valued $A$-functors. In particular, it will follow that many examples of $X$-functors are coherent. However, the reader interested only in the Picard group results may ignore the next result and its corollaries, since for purposes of the finiteness result (7.1), it is sufficient to know that a given $X$-functor is locally coherent.

Theorem 5.6. Assume that $X$ is separated. Let $F$ be a locally module-quasicoherent [resp. locally module-coherent] $X$-functor. Then $F$ is module-quasicoherent [resp. module-coherent].

Proof. In the course of the proof we refer to sheaves, which shall always mean sheaves for the Zariski topology. We work not with $X$-functors, but with functors whose source is $\langle\langle X \text {-schemes which are quasi-compact }\rangle\rangle^{\circ}$, as discussed briefly at the beginning of this section.

Let $U_{1}, \ldots, U_{n}$ be as in the definition of locally module-quasi-coherent (or locally module-coherent). By (5.4), we may assume that each $U_{i}$ is affine. Since $X$ is separated, it follows that the open subschemes $U_{i} \cap U_{j}$ are affine and that the inclusions of $U_{i}$ in $X$ and of $U_{i} \cap U_{j}$ in $X$ are affine morphisms.

First we prove the module-quasi-coherent case. (This will be needed for the module-coherent case.) Regard $\left.F\right|_{U_{i}}$ and $\left.F\right|_{U_{i} \cap U_{j}}$ as module-valued $X$-functors. It follows from (5.5) and (5.4) that these are module-quasi-coherent. Because $F$ is a sheaf, we have a left exact sequence

$$
\left.\left.0 \rightarrow F \rightarrow \prod_{i=1}^{n} F\right|_{U_{i}} \rightarrow \prod_{1 \leq i<j \leq n} F\right|_{U_{i} \cap U_{j}}
$$

of module-valued $X$-functors. Hence $F$ is module-quasi-coherent.

Now we show that if $F$ is a module-quasi-coherent $X$-functor, then there exists a morphism $\phi: \mathcal{M} \rightarrow \mathcal{N}$ of quasi-coherent $\mathcal{O}_{X}$-modules such that $F \cong \underline{\operatorname{Ker}}(\phi)$. By an unstated analog of (2.2), we may embed $F$ as a sub-module-valued-functor of $\underline{\mathcal{M}}$ for some quasi-coherent $\mathcal{O}_{X}$-module $\mathcal{M}$. Let $G=(\underline{\mathcal{M}} / F)^{*}$, where the superscript * denotes sheafification. By (3.5), it follows that $G$ is locally module-quasi-coherent, so (by the first part of the proof) $G$ is module-quasi-coherent. Embed $G$ as a submodule-valued-functor of $\underline{\mathcal{N}}$ for some quasi-coherent $\mathcal{O}_{X}$-module $\mathcal{N}$. Let $\phi: \mathcal{M} \rightarrow$ $\mathcal{N}$ be the induced map. Then $F=\underline{\operatorname{Ker}}(\phi)$, as required.

Now we begin the proof of the module-coherent case. By what we have already shown, we may assume that there exists a morphism $\phi: \mathcal{M} \rightarrow \mathcal{N}$ of quasi-coherent $\mathcal{O}_{X}$-modules such that $F=\underline{\operatorname{Ker}}(\phi)$. We will show that there exist coherent sub$\mathcal{O}_{X}$-modules $\mathcal{M}_{0} \subset \mathcal{M}_{1} \subset \mathcal{M}$ and $\mathcal{N}_{0} \subset \mathcal{N}$ such that $\phi\left(\mathcal{M}_{0}\right) \subset \mathcal{N}_{0}$ and such that 
in the induced diagram

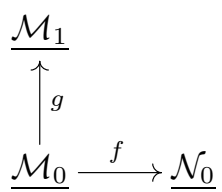

we have $F \cong g[\operatorname{ker}(f)]^{*}$.

Let us verify that the construction of this data will complete the proof. We must show that $g[\operatorname{ker}(f)]^{*}$ is module-coherent. Let $\mathcal{P}$ denote the co-fiber product of $\mathcal{M}_{1}$ with $\mathcal{N}_{0}$ over $\mathcal{M}_{0}$, taken in the category of quasi-coherent $\mathcal{O}_{X}$-modules. Then in fact the induced diagram

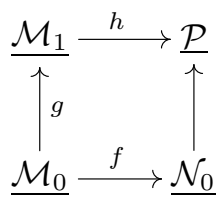

is cocartesian, if it is viewed as a diagram in

$\langle\langle$ module-valued $X$-functors which are sheaves $\rangle\rangle$.

Since we have $\operatorname{ker}(h) \cong g[\operatorname{ker}(f)]^{*}$, the theorem will follow.

It remains to construct the data. Certainly, for any choice of data, there is a canonical morphism

$$
\psi: g[\operatorname{ker}(f)]^{*} \rightarrow F
$$

of module-valued $X$-functors.

We work on choosing $\mathcal{M}_{0}$. Let $\left\{\mathcal{M}_{\lambda}\right\}_{\lambda \in \Lambda}$ be the coherent sub- $\mathcal{O}_{X}$-modules of $\mathcal{M}$. Let $H_{\lambda}$ be the sheafified image of the map $\mathcal{M}_{\lambda} \rightarrow \underline{\mathcal{M}}$. Then the $H_{\lambda}$ form a directed

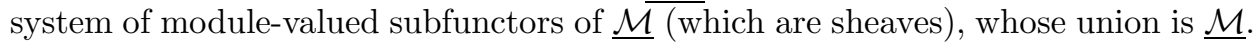
(The validity of the last assertion depends on the simplifying assumption made in the first paragraph of this proof, to the effect that we work only with quasi-compact $X$-schemes.) Let $F_{\lambda}=F \cap H_{\lambda}$. Then the $F_{\lambda}$ form a directed system of modulevalued subfunctors of $F$ (which are sheaves), whose union is $F$. Then it follows that (3.8) that $F=F_{\lambda}$ for some $\lambda \in \Lambda$. Let $\mathcal{M}_{0}=\mathcal{M}_{\lambda}$. Then $F$ is contained in the sheafified image of the map $\underline{\mathcal{M}_{0}} \rightarrow \underline{\mathcal{M}}$.

Now we work on choosing $\overline{\mathcal{N}_{0}}$. Let $\left\{\mathcal{N}_{\lambda}\right\}_{\lambda \in \Lambda}$ be the coherent sub- $\mathcal{O}_{X^{-}}$ modules of $\mathcal{N}$ which contain $\phi\left(\mathcal{M}_{0}\right)$. Let $I_{\lambda}$ be the sheafified image of the map $\operatorname{ker}\left[\mathcal{M}_{0} \rightarrow \mathcal{N}_{\lambda}\right] \rightarrow \underline{\mathcal{M}}$. Then the $I_{\lambda}$ form a directed system of module-valued subfunctors of $\bar{F}$ (which are sheaves). We will show that the union of the $I_{\lambda}$ is $F$. Let $J_{\lambda}=\operatorname{ker}\left[\underline{\mathcal{M}_{0}} \rightarrow \underline{\mathcal{N}_{\lambda}}\right]$. It suffices to show that $\operatorname{ker}\left[\underline{\mathcal{M}_{0}} \rightarrow \underline{\mathcal{N}}\right]$ is the union of the $J_{\lambda}$. Let $Y$ be a quasi-compact $X$-scheme. Let $\alpha \in \Gamma\left[\left(\mathcal{M}_{0}\right)_{Y}\right]$, and assume that $\alpha \mapsto 0$ in $\Gamma\left(\mathcal{N}_{Y}\right)$. We must show that there exists some $\mathcal{N}_{\lambda}$ such that $\alpha \mapsto 0$ in $\Gamma\left[\left(\mathcal{N}_{\lambda}\right)_{Y}\right]$. By ([19], I.6.9.9), we know that $\mathcal{N}$ is the direct limit of the $\mathcal{N}_{\lambda}$. It follows that $\mathcal{N}_{Y}$ is the direct limit of the $\left(\mathcal{N}_{\lambda}\right)_{Y}$. This implies the statement about $\alpha \mapsto 0$, and hence that the union of the $I_{\lambda}$ is $F$. Arguing as in the preceding paragraph, we see that for some $\lambda \in \Lambda$, we have $I_{\lambda}=F$. Let $\mathcal{N}_{0}=\mathcal{N}_{\lambda}$. It follows now that no matter how we choose $\mathcal{M}_{1}$, the map $\psi$ will be an epimorphism, when viewed as a morphism in the category of module-valued $X$-functors which are sheaves. 
Now we work on choosing $\mathcal{M}_{1}$. Let $G=\operatorname{ker}(f)$. Then $G$ is module-coherent. Let $K=\operatorname{ker}[G \rightarrow \underline{\mathcal{M}}]$. Then $K=\operatorname{ker}[G \rightarrow F]$, so $K$ is locally module-coherent. Let $\left\{\mathcal{M}_{\lambda}\right\}_{\lambda \in \Lambda}$ be the coherent sub- $\mathcal{O}_{X}$-modules of $\mathcal{M}$ which contain $\mathcal{M}_{0}$. (These $\mathcal{M}_{\lambda}$ are not the same as those defined earlier.) Let $K_{\lambda}=\operatorname{ker}\left[G \rightarrow \mathcal{M}_{\lambda}\right]$. Then the $K_{\lambda}$ form a directed system of module-valued subfunctors of $K$ (which are sheaves). Arguing as in the preceding paragraph, we see that the union of the $K_{\lambda}$ is $K$. But $K$ is locally module-coherent, so it follows from (3.8) that $K_{\lambda}=K$ for some $\lambda \in \Lambda$. Let $\mathcal{M}_{1}=\mathcal{M}_{\lambda}$. Then $\psi$ is a monomorphism. Hence $\psi$ is an isomorphism.

Corollary 5.7. Assume that $X$ is separated. Let $\phi: F \rightarrow G$ be a morphism of module-coherent $X$-functors. Then the Zariski sheaf associated to $\operatorname{Coker}(\phi)$ is module-coherent.

Corollary 5.8. Assume that $X$ is separated. Then the category of module-coherent $X$-functors is abelian.

Corollary 5.9. Assume that $X$ is separated. Let $F$ be a module-coherent $X$ functor. Then $F$ has level $\leq 1$. That is, there exists a left exact sequence

$$
0 \rightarrow F \rightarrow \underline{\mathcal{M}} \rightarrow \underline{\mathcal{N}}
$$

in which $\mathcal{M}$ and $\mathcal{N}$ are coherent $\mathcal{O}_{X}$-modules.

The constructions Hom, Aut and so forth which we defined in $\S 4$ make sense for $X$-functors. For example, if $F$ and $G$ are module-valued $X$-functors, we let $\operatorname{Hom}(F, G)$ denote the $X$-functor given by

$$
Y \mapsto \operatorname{Hom}_{\text {module-valued } Y \text {-functors }}\left(\left.F\right|_{Y},\left.G\right|_{Y}\right) \text {. }
$$

Proposition 5.10. Assume that $X$ is separated. Let $F$ and $G$ be module-coherent $X$-functors. Then $\operatorname{Hom}(F, G)$ is module-coherent.

Proof. By (4.7) and (5.6), it suffices to show that $\operatorname{Hom}(F, G)$ is a Zariski sheaf. This can be directly checked from the definition.

Similarly, we have:

Corollary 5.11. Assume that $X$ is separated. Then for any module-coherent $X$ functor $F, \operatorname{End}(F)$ is module-coherent, and $\operatorname{Aut}(F)$ is coherent. Let $R$ and $S$ be module-coherent, algebra-valued $X$-functors. Then $\mathbf{H o m}_{\text {alg }}(R, S)$ and $\mathbf{A u t}_{\text {alg }}(R)$ are coherent.

Now we consider the extent to which a module-quasi-coherent $A$-functor can be viewed as a direct limit of module-coherent $A$-functors. These considerations will enter into an analysis of extensions of module-coherent $X$-functors, which will be the last topic discussed in this section.

Unfortunately, it is not the case that every module-quasi-coherent $A$-functor $H$ is the direct limit of its module-coherent subfunctors. For an example, let $H=\operatorname{Im}(\varphi)$, where $\varphi$ is as in (3.9). If there existed a directed system $\left\{H_{\lambda}\right\}_{\lambda \in \Lambda}$ of modulecoherent subfunctors of $H$, with union $H$, it would follow by (3.8), applied with $F=\underline{\mathbb{Z}}$ and $F_{\lambda}=\varphi^{-1}\left(H_{\lambda}\right)$, that $\varphi$ factors through a module-coherent subfunctor of $\underline{\mathbb{Q}}$, and hence that $\operatorname{ker}(\varphi)$ is module-coherent, which is not the case.

Definition. A module-valued $A$-functor $C$ is bar-module-coherent if it is modulequasi-coherent and if there exists a module-coherent $A$-functor $F$, together with an epimorphism $F \rightarrow C$. 
A bar-module-coherent $A$-functor need not be module-coherent. For an example, let $H=\operatorname{Im}(\varphi)$, where $\varphi$ is as in (3.9). Then $H$ is bar-module-coherent, but not module-coherent, since otherwise $\operatorname{ker}(\varphi)$ would be module-coherent.

Lemma 5.12. Let $F$ be a module-quasi-coherent A-functor. Then there exists a directed system $\left\{F_{\lambda}\right\}_{\lambda \in \Lambda}$ of bar-module-coherent subfunctors of $F$, with union $F$.

Proof. Choose $A$-modules $M, N$ and a left exact sequence

$$
0 \rightarrow F \rightarrow \underline{M} \stackrel{h}{\rightarrow} \underline{N}
$$

of module-valued $A$-functors, in which $h$ is induced by a homomorphism $\phi: M \rightarrow N$ of $A$-modules. Let $\mathcal{S}$ denote the collection $\left\{\left(M_{\lambda}, N_{\lambda}\right)\right\}_{\lambda \in \Lambda}$ consisting of all pairs $\left(M_{\lambda}, N_{\lambda}\right)$ in which $M_{\lambda}$ is a finitely generated submodule of $M, N_{\lambda}$ is a finitely generated submodule of $N$, and $\phi\left(M_{\lambda}\right) \subset N_{\lambda}$. For each $\lambda \in \Lambda$, let $h_{\lambda}: \underline{M_{\lambda}} \rightarrow \underline{N_{\lambda}}$ be the induced morphism of $A$-functors. Let $K_{\lambda}=\operatorname{ker}\left(h_{\lambda}\right)$. Then $K_{\lambda}$ is modulecoherent. There is a canonical map $f_{\lambda}: K_{\lambda} \rightarrow F$. Let $F_{\lambda}=\operatorname{Im}\left(f_{\lambda}\right)$. Then $F_{\lambda}$ is bar-module-coherent, and the $F_{\lambda}$ form a directed system of subfunctors of $F$.

Let $B$ be a commutative $A$-algebra, and let $c \in F(B)$. Then $c \in M_{B}$. Choose a finitely generated submodule $M_{\lambda} \subset M$ and an element $c_{\lambda} \in\left(M_{\lambda}\right)_{B}$ such that $c_{\lambda} \mapsto c$. There exists a finitely generated submodule $N_{\lambda}$ of $N$ such that $\phi\left(M_{\lambda}\right) \subset N_{\lambda}$ and such that $c_{\lambda} \mapsto 0$ in $\left(N_{\lambda}\right)_{B}$. It follows that $\bigcup_{\lambda \in \Lambda} F_{\lambda}=F$.

We close this section with some questions and a result about extensions:

Problem 5.13. Let

$$
1 \rightarrow F^{\prime} \rightarrow F \rightarrow F^{\prime \prime} \rightarrow 1
$$

be a short exact sequence of group-valued $X$-functors. Assume that $F^{\prime}$ and $F^{\prime \prime}$ are coherent. Is F coherent?

Problem 5.14. Let

$$
0 \rightarrow F^{\prime} \rightarrow F \rightarrow F^{\prime \prime} \rightarrow 0
$$

be a short exact sequence of module-valued $X$-functors. Assume that $F^{\prime}$ and $F^{\prime \prime}$ are module-coherent. Is F module-coherent?

We can prove this if we assume that $F$ is module-quasi-coherent and that $X$ is separated:

Proposition 5.15. Assume that $X$ is separated. Let

$$
0 \rightarrow F^{\prime} \rightarrow F \rightarrow F^{\prime \prime} \rightarrow 0
$$

be a short exact sequence of module-valued $X$-functors. Assume that $F^{\prime}$ and $F^{\prime \prime}$ are module-coherent. Assume that $F$ is module-quasi-coherent. Then $F$ is modulecoherent.

Proof. By (5.6), we may reduce to working with $A$-functors. By (5.12), $F$ is the direct limit of its bar-module-coherent subfunctors. By (3.8), it follows that there exists a bar-module-coherent subfunctor $B$ of $F$ such that $B$ maps onto $F^{\prime \prime}$. Since $F^{\prime}$ is module-coherent, we see that $F$ is itself bar-module-coherent. Choose a modulecoherent $A$-functor $C$ and an epimorphism $\beta: C \rightarrow F$. 
Let $P$ be the fiber product of $F^{\prime}$ and $C$ over $F$. Then we have a commutative diagram with exact rows:

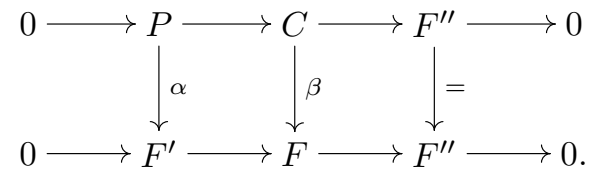

Hence $\operatorname{ker}(\alpha) \cong \operatorname{ker}(\beta)$. Since $C$ and $F^{\prime \prime}$ are module-coherent, so is $P$. Since $F^{\prime}$ and $P$ are module-coherent, so is $\operatorname{ker}(\alpha)$. Hence $\operatorname{ker}(\beta)$ is module-coherent. Since $\operatorname{ker}(\beta)$ and $C$ are module-coherent, it follows by $(2.7)$ that $F$ is also module-coherent.

This fact will be used in the proof of (10.2).

\section{Continuity}

We consider the extent to which quasi-coherent $A$-functors preserve limits, and briefly, the extent to which they preserve colimits. We prove that a module-quasicoherent $A$-functor which preserves products is module-coherent. Although these topics do not play much of a role in the subsequent parts of this paper, they are very natural. Some important examples of limit and colimit preservation which have arisen previously are Grothendieck's theorem on formal functions (see e.g., [21], III:11), and Grothendieck's notion of functors which are locally of finite presentation ([1], 1.5), which enters into Artin's criterion for representability ([3], 3.4).

We begin by recalling some definitions about continuity of functors. Let $\mathcal{C}$ and $\mathcal{A}$ be complete (meaning small-complete) categories, and let $F: \mathcal{C} \rightarrow \mathcal{A}$ be any functor. Then $F$ is continuous if it preserves limits, i.e. if for every small category $\mathcal{D}$, and every functor $H: \mathcal{D} \rightarrow \mathcal{C}$, the canonical map

$$
F\left(\varliminf_{\lim } H\right) \rightarrow \varliminf_{\lim }(F \circ H)
$$

is an isomorphism. (See, e.g., [26], V.4.) It is also of interest to know if $F$ preserves more restricted sorts of limits, e.g. does it preserve arbitrary products, or does it preserve equalizers. These conditions correspond to placing appropriate restrictions on $\mathcal{D}$.

For particular sorts of limits, one can usually rephrase the continuity condition in a simpler way. For example, $F$ preserves products if and only if for every set $\left\{X_{i}\right\}_{i \in I}$ of objects in $\mathcal{C}$, the canonical map

$$
F\left(\prod_{i \in I} X_{i}\right) \rightarrow \prod_{i \in I} F\left(X_{i}\right)
$$

is an isomorphism.

Just as one can check a category for completeness by checking if it has products and equalizers, so one can check a functor for continuity by checking if it preserves products and equalizers.

We will study the continuity properties of $A$-functors. There are two general observations to be made. The first observation is that an (abelian group)-valued $A$-functor or a module-valued $A$-functor is continuous (or preserves a particular kind of limit) if and only if the same statement holds for the underlying functor from $\langle\langle$ commutative $A$-algebras $\rangle\rangle$ to $\langle\langle$ sets $\rangle\rangle$. The second observation is the following lemma, whose proof is left to the reader: 
Lemma 6.1. Let $M$ be an A-module and let

$$
F \longmapsto G \rightarrow \underline{M}
$$

be a left exact sequence of $A$-functors. If $\underline{M}$ and $G$ preserve a particular type of limit, then so does $F$.

The phrase "particular type of limit" is to be construed as referring to a class of limits which is constrained by some restriction on the categories $\mathcal{D}$ and/or the functors $H: \mathcal{D} \rightarrow\langle\langle$ commutative $A$-algebras $\rangle\rangle$ which are allowed.

We proceed to investigate the extent to which quasi-coherent $A$-functors preserve various types of limits. First we consider finite limits, that is, limits in which the category $\mathcal{D}$ has only finitely many objects and morphisms. The most important examples are finite products (including terminal objects), and equalizers. Also, if a functor preserves finite products and equalizers, then it preserves all finite limits. As for finite products, one sees easily (using (6.1)) that:

Proposition 6.2. Let $F$ be a quasi-coherent A-functor. Then $F$ preserves finite products.

Now we consider equalizers. An $A$-module $M$ is flat if and only if the functor $\otimes_{A} M:\langle\langle A$-modules $\rangle\rangle \rightarrow\langle\langle A$-modules $\rangle\rangle$ preserves equalizers, so the analogous fact for $A$-functors is hardly surprising:

Proposition 6.3. Let $M$ be an A-module. Then the A-functor $M$ preserves equalizers if and only if $M$ is flat.

Proof. The case where $M$ is flat is left to the reader. So suppose that $M$ is not flat. Then (see, e.g., [27], 3.53) there exists an ideal $I \subset A$ such that the induced map $\phi: M \otimes_{A} I \rightarrow M$ is not injective. Let $y \in \operatorname{ker}(\phi)-\{0\}$. Let $a_{1}, \ldots, a_{n}$ be generators for $I$. Choose $m_{1}, \ldots, m_{n} \in M$ such that $y=\sum_{i=1}^{n} m_{i} \otimes a_{i}$. Let $B=A\left[x_{1}, \ldots, x_{n}\right] /\left(\left\{x_{i} x_{j}\right\}_{1 \leq i, j \leq n}\right)$. Let $f, g: B \rightarrow A$ be the $A$-algebra maps given by $f\left(x_{i}\right)=a_{i}$ and $g\left(x_{i}\right)=0$ for each $i$. Let $p \in M \otimes_{A} B$ be $\sum_{i=1}^{n} m_{i} \otimes x_{i}$. Then $\left(f \otimes_{A} M\right)(p)=\left(g \otimes_{A} M\right)(p)$. Let $\operatorname{Eq}(f, g)$ be the equalizer of $f$ and $g$. Since $y \neq 0$, it follows (after a little work) that $p$ does not lie in the image of the canonical map $\lambda: \operatorname{Eq}(f, g) \otimes_{A} M \rightarrow \operatorname{Eq}\left(f \otimes_{A} M, g \otimes_{A} M\right)$, and hence that $\lambda$ is not an isomorphism. Hence $\underline{M}$ does not preserve the equalizer of $f$ and $g$.

It follows that $\underline{M}$ preserves finite limits if and only if $M$ is flat. In addition, (6.1) yields the following corollary:

Corollary 6.4. Any quasi-coherent A-functor which is built up from flat modules will preserve finite limits.

It is worth noting that if $F$ is an $A$-functor which preserves finite limits, then $F$ is a sheaf with respect to the fppf topology; indeed the sheaf axioms may be viewed as a statement about continuity. While quasi-coherent $A$-functors do not always preserve finite limits, we do know that they are sheaves with respect to the fppf topology.

Remark 6.5. It is not difficult to see that any coherent $A$-functor preserves products. Any coherent $A$-functor which is built up from finitely generated projective $A$ modules will also preserve equalizers, and hence all limits. Of course, the $A$-functors which are built up in this way are exactly the $A$-functors which are representable by an $A$-algebra of finite type. (One might call these affine representable.) 
In general, coherent $A$-functors do not preserve inverse limits. For example, if $A=\mathbb{Z}$, then the $A$-functor $\mathbb{Z} / 2 \mathbb{Z}$ does not preserve the limit of

$$
\mathbb{Z}[x] /\left(x^{2}\right) \stackrel{x \mapsto 3 x}{\leftarrow} \mathbb{Z}[x] /\left(x^{2}\right) \stackrel{x \mapsto 3 x}{\longleftarrow} \cdots .
$$

The transition maps here are not surjective. One might hope that the limit would be preserved if the transition maps were surjective, or at least if the system satisfied the Mittag-Leffler condition ([16], 0:13.1). Unfortunately, this is not the case:

Example 6.6. Let $A=\mathbb{Z}$. Let

$$
B_{n}=\mathbb{Z}\left[x_{1}, x_{2}, \ldots, y, z_{1}, \ldots, z_{n-1}\right] /\left(2 x_{1}, 2 x_{2}, \ldots, Q\right),
$$

where $Q$ denotes the set of homogeneous quadratic polynomials in all of the given variables. Form an inverse system of commutative $A$-algebras

$$
B_{1} \leftarrow B_{2} \leftarrow B_{3} \leftarrow \cdots
$$

in which (for each $n>1$ ) the transition map $B_{n} \rightarrow B_{n-1}$ is given by $x_{k} \mapsto x_{k+1}$, $y \mapsto x_{1}+y, z_{1} \mapsto x_{1}, z_{k} \mapsto z_{k-1}$ for $k \geq 2$. The transition maps of this system are surjective.

The elements $2 y, 2 y, \ldots$ form a coherent sequence. Each element in this sequence is divisible by 2 , but there is no coherent sequence which when multiplied by 2 yields $2 y, 2 y, \ldots$. Hence the $A$-functor $\underline{\mathbb{Z} / 2 \mathbb{Z}}$ does not preserve the limit of this system.

From ([4], 10.13) and (6.1) it follows that:

Proposition 6.7. Let $F$ be a coherent A-functor. Let $B$ be a commutative noetherian A-algebra. Let $I \subset B$ be an ideal. Then $F$ preserves the limit of

$$
B / I \leftarrow B / I^{2} \leftarrow B / I^{3} \cdots .
$$

There may well be interesting situations in which coherent functors preserve inverse limits, other than those given in (6.7) and (6.5).

Figure 1 summarizes what we know about continuity properties of certain kinds of quasi-coherent $A$-functors. For precise statements, see (6.2), (6.4), (6.5), and (6.7).

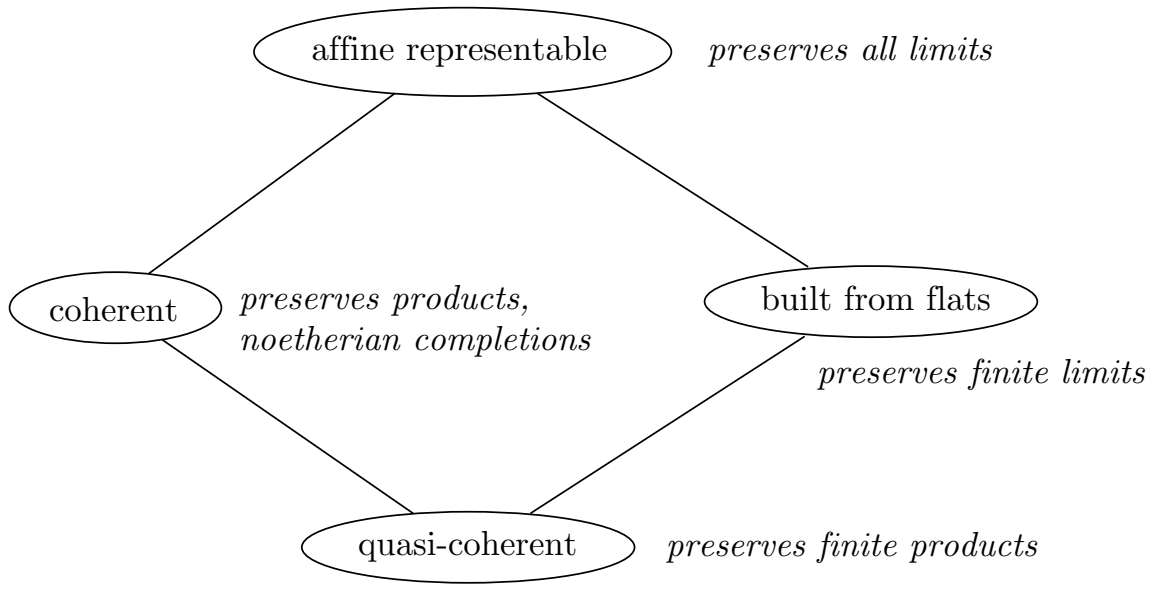

Figure 1 
Our next objective is to show that a module-quasi-coherent $A$-functor which preserves products is module-coherent. This as well as (6.3) allows one to use knowledge about continuity to deduce some information about how a quasi-coherent $A$-functor is built up.

Lemma 6.8. Let $L_{1}$ and $L_{2}$ be module-quasi-coherent subfunctors of a modulequasi-coherent $A$-functor $H$. If $L_{1}(B) \subset L_{2}(B)$ for every finitely generated commutative A-algebra $B$, then $L_{1} \subset L_{2}$.

Proof. Let $G=L_{1} /\left(L_{1} \cap L_{2}\right)$. Since $G$ is module-quasi-coherent, we have $G \cong$ $\underline{\operatorname{Ker}}(f)$ for some homomorphism $f: M \rightarrow N$ of $A$-modules. Then $\operatorname{Ker}\left(f \otimes_{A} B\right)=0$ for every finitely generated commutative $A$-algebra $B$, from which it follows that $\operatorname{Ker}\left(f \otimes_{A} B\right)=0$ for every commutative $A$-algebra $B$. Hence $G=0$.

Lemma 6.9. Let $\phi: H \rightarrow N$ be a homomorphism of A-modules. Assume that $H$ is finitely generated. Let $B$ be a commutative $A$-algebra. Let $h \in \operatorname{Ker}\left(\phi \otimes_{A} B\right)$. Then there exists a finitely generated submodule $N_{0}$ of $N$ such that $\phi$ factors through $N_{0}$ and such that $h \mapsto 0$ in $N_{0} \otimes_{A} B$.

Proof. Certainly $N$ is the direct limit of its finitely generated submodules which contain $\phi(H)$. The statement follows from the fact that tensor products commute with direct limits.

Proposition 6.10. Let $F$ be a module-quasi-coherent A-functor which preserves products. Then $F$ is module-coherent.

Proof. All tensor products in this proof are over $A$. We may assume $F=\underline{\operatorname{Ker}}(f)$ for some homomorphism $f: M \rightarrow N$ of $A$-modules. We will show that there exists a finitely generated submodule $L \subset M$ such that if $i: L \rightarrow M$ is the inclusion, then $\underline{\operatorname{Ker}}(f) \subset \underline{\operatorname{Im}}(i)$. Choose a complete set of isomorphism class representatives $\left\{B_{\lambda}\right\}_{\lambda \in \Lambda}$ for the finitely generated commutative $A$-algebras. Form the disjoint union $T=\coprod_{\lambda \in \Lambda} \operatorname{Ker}\left(f \otimes B_{\lambda}\right)$. For any $t \in T$, let $\lambda(t)$ denote the corresponding element of $\Lambda$. Let $B=\prod_{t \in T} B_{\lambda(t)}$. The elements $t \in T$ define an element $x \in$ $\prod_{t \in T} \operatorname{Ker}\left(f \otimes B_{\lambda(t)}\right)$. Since $F$ preserves products, $x \in \operatorname{Ker}(f \otimes B)$. Write $x=$ $\sum_{j=1}^{r} m_{j} \otimes b_{j}$, where $m_{1}, \ldots, m_{r} \in M, b_{1}, \ldots, b_{r} \in B$. Let $L$ be the submodule of $M$ generated by $m_{1}, \ldots, m_{r}$. The expansion of $x$ defines an element $\tilde{x} \in L \otimes B$ with the property that $\tilde{x} \mapsto x$. From this it follows that $\operatorname{Ker}\left(f \otimes B_{\lambda}\right) \subset \operatorname{Im}\left(i \otimes B_{\lambda}\right)$ for every $\lambda \in \Lambda$. By (6.8), we have $\underline{\operatorname{Ker}}(f) \subset \underline{\operatorname{Im}}(i)$.

Since $\tilde{x} \mapsto 0$ in $N \otimes B$, it follows from (6.9) that there exists a finitely generated submodule $N_{0}$ of $N$ such that $L \rightarrow N$ factors through $N_{0}$ and such that $\tilde{x} \mapsto 0$ in $N_{0} \otimes B$.

For any $t \in T$, let $\lambda=\lambda(t)$, and let $\tilde{t} \in L \otimes B_{\lambda}$ be the image of $\tilde{x}$ under the canonical map $\pi_{t}: L \otimes B \rightarrow L \otimes B_{\lambda}$ which projects onto the $t$ th factor. Then $\left(i \otimes B_{\lambda}\right)(\tilde{t})=t$, and $\tilde{t} \mapsto 0$ in $N_{0} \otimes B_{\lambda}$. Let $\underline{K}=\underline{\operatorname{Ker}}\left(L \rightarrow N_{0}\right)$. Then $\underline{K}\left(B_{\lambda}\right)$ maps onto $F\left(B_{\lambda}\right)$. By (6.8), the map $\psi: \underline{K} \rightarrow F$ is an epimorphism. In particular, $F$ is the image of a module-coherent $A$-functor.

Let $Q=\operatorname{Ker}(\psi)$. Since $\underline{K}$ is module-coherent, it preserves products. Since $F$ also preserves products, it follows by (6.1) that $Q$ preserves products. Replaying the first part of the proof, with $F$ replaced by $Q$, we see that $Q$ is also the image of a module-coherent $A$-functor. Hence $F$ is the cokernel of a map of module-coherent $A$-functors, so $F$ is module-coherent. 
The last objective of this section is to consider (briefly) the extent to which coherent functors preserve colimits.

Whether or not coherent functors preserve finite colimits and coproducts does not seem to be an interesting question. One reason for this is that the forgetful functor from $\langle\langle$ groups $\rangle\rangle$ to $\langle\langle$ sets $\rangle\rangle$ does not preserve coproducts or finite colimits. (One can substitute various other categories for $\langle\langle$ groups $\rangle\rangle$ with the same outcome.) Therefore a functor from $\langle\langle$ commutative $A$-algebras $\rangle\rangle$ to $\langle\langle$ groups $\rangle\rangle$ might preserve such colimits, but the induced functor from $\langle\langle$ commutative $A$-algebras $\rangle\rangle$ to $\langle\langle$ sets $\rangle\rangle$ might not. With either interpretation, preservation of coproducts or finite colimits seems like a bizarre requirement.

On the other hand, the forgetful functor $\langle\langle$ groups $\rangle\rangle \rightarrow\langle\langle$ sets $\rangle\rangle$ does preserve direct limits, ${ }^{1}$ and the same statement is valid with various other categories substituted for $\langle\langle$ groups $\rangle\rangle$. Therefore, the situation for direct limits is just like the situation which holds for all limits: an (abelian group)-valued $A$-functor or a modulevalued $A$-functor preserves direct limits if and only if the same statement holds for the underlying functor from $\langle\langle$ commutative $A$-algebras $\rangle\rangle$ to $\langle\langle$ sets $\rangle\rangle$.

The analog of (6.1) for direct limits is valid, and since tensor products commute with direct limits, it follows that any quasi-coherent $A$-functor preserves direct limits. Artin remarks that nearly all $A$-functors which occur in practice do this; in Artin's terminology an $A$-functor which preserves direct limits is said to be locally of finite presentation ([1], 1.5). This condition enters into his criterion for representability ([3], 3.4).

\section{Global Sections}

Let us say that a group is linear if it may be embedded as a subgroup of $\mathrm{GL}_{n}\left(k_{1}\right) \times \cdots \times \mathrm{GL}\left(k_{r}\right)$ for some $n$ and some fields $k_{1}, \ldots, k_{r}$. We shall want to have some control over the fields: a group is $X$-linear if there exist points $x_{1}, \ldots, x_{r} \in X$ (not necessarily closed) and finitely generated field extensions $k_{i}$ of $k\left(x_{i}\right)$ (for each $i$ ) such that the group may be embedded as a subgroup of $\mathrm{GL}_{n}\left(k_{1}\right) \times \cdots \times \mathrm{GL}_{n}\left(k_{r}\right)$ for some $n$.

The purpose of this section is two-fold. The first purpose is to develop a tool (the next theorem) for proving that groups are $X$-linear. The second purpose is to study the torsion in $X$-linear groups. Our result is (7.2), or in a slightly different form (7.4).

Theorem 7.1. Let $G$ be a group-valued locally coherent $X$-functor. Then $G(X)$ is $X$-linear.

Before proving this theorem, we will study the torsion in $X$-linear groups. For any abelian group $H$, one can try to determine for which $n \in \mathbb{N}$ one has $\left|{ }_{n} H\right|<\infty$. If $n=p_{1}^{k_{1}} \cdots p_{r}^{k_{r}}$, where $p_{1}, \ldots, p_{r}$ are prime numbers, then $\left|{ }_{n} H\right|<\infty$ if and only if $\left|p_{i} H\right|<\infty$ for each $i$. Therefore we may as well restrict to the problem of determining when $\left|{ }_{p} H\right|<\infty$, where $p$ is prime.

If $C$ is a commutative ring, let us say that a morphism $\pi: X \rightarrow \operatorname{Spec}(C)$ is essentially of finite type if there exist a commutative ring $D$, a homomorphism $\phi: C \rightarrow D$ which is essentially of finite type, and a morphism of finite type $\pi_{0}: X \rightarrow \operatorname{Spec}(D)$, such that $\pi=\operatorname{Spec}(\phi) \circ \pi_{0}$. Note that if $X$ is essentially of

\footnotetext{
${ }^{1}$ The reader is reminded that direct limits are a type of colimit.
} 
finite type over $\mathbb{Z}$, and $x \in X$, then $k(x)$ is a finitely generated field extension of its prime subfield.

Proposition 7.2. Let $H$ be an $X$-linear abelian group.

(a) There are only finitely many prime numbers $p$ such that $H$ has infinite $p$ torsion. Moreover, such a $p$ cannot be invertible in $\Gamma\left(X, \mathcal{O}_{X}\right)$.

(b) If $X$ is essentially of finite type over $\mathbb{Z}$ or over $\mathbb{Z}_{p}$ (for some prime number $p)$, then there exist prime numbers $p_{1}, \ldots, p_{n}$, none of which are invertible in $\Gamma\left(X, \mathcal{O}_{X}\right)$, such that the subgroup of $H$ consisting of torsion prime to $p_{1} \cdots p_{n}$ is finite.

Clearly, if in the above proposition, $X$ is essentially of finite type over $\mathbb{Z}_{p}$, then the list of primes $p_{1}, \ldots, p_{n}$ may be taken to be the single prime $p$. Also we have:

Corollary 7.3. Let $H$ be an $X$-linear abelian group. If $X$ is essentially of finite type over $\mathbb{Q}$ or over $\mathbb{Q}_{p}$ (for some prime number $p$ ), then the torsion subgroup of $H$ is finite.

We now state a generalization of the proposition to the nonabelian case:

Proposition 7.4. Let $H$ be an $X$-linear group.

(a) Let $n \in \mathbb{N}$ be invertible in $\Gamma\left(X, \mathcal{O}_{X}\right)$. Then there exists some $N \in \mathbb{N}$ such that whenever $K$ is an $n$-torsion abelian subgroup of $H$, we have $|K| \leq N$.

(b) If $X$ is essentially of finite type over $\mathbb{Z}$ or over $\mathbb{Z}_{p}$ (for some prime number $p)$, then there exist prime numbers $p_{1}, \ldots, p_{n}$, none of which are invertible in $\Gamma\left(X, \mathcal{O}_{X}\right)$, and some $N \in \mathbb{N}$, such that if $K$ is an abelian subgroup of $H$, and every element of $K$ is torsion prime to $p_{1} \cdots p_{n}$, then $|K| \leq N$.

Lemma 7.5. Let $(A, \mathfrak{m}, k)$ be a local ring. Let $n \in \mathbb{N}$, and assume that $n$ is invertible in $A$. Then the canonical map:

$$
\{n \text {th roots of unity in } A\} \rightarrow\{n \text {th roots of unity in } k\}
$$

is injective.

Proof. Let $n \in \mathbb{N}, x \in A, a \in \mathfrak{m}$, and suppose that $x^{n}=(x+a)^{n}=1$. Then $0=\left[(x+a)^{n}-x^{n}\right]=a\left[n x^{n-1}+c\right]$, for some $c \in \mathfrak{m}$. But $n x^{n-1}$ is a unit, so $n x^{n-1}+c$ is a unit, so $a=0$.

It is known ([10], $\S 14, \# 7$, Corollary 2 to Proposition 17) that a field finitely generated over its prime subfield (as a field extension) contains only finitely many roots of unity. This also holds for a field finitely generated over $\mathbb{Q}_{p}$. We will need a modest generalization of these statements:

Lemma 7.6. Let $K$ be a finitely generated field extension of $F$, where $F$ is $\mathbb{Q}$, or $\mathbb{F}_{p}$, or $\mathbb{Q}_{p}$, for some prime number $p$. Then there exists a constant $c$ such that for every $m \in \mathbb{N}$, and every finite field extension $L$ of $K$ with $[L: K] \leq m$, the number of roots of unity in $L$ is $\leq$ cm if $F=\mathbb{Q}$, and is $\leq c^{m}$ if $F$ is $\mathbb{F}_{p}$ or $\mathbb{Q}_{p}$.

Proof. Let $x_{1}, \ldots, x_{r}$ be a transcendence basis for $K$ over $F$. Let $s=$ $\left[K: F\left(x_{1}, \ldots, x_{r}\right)\right]$. If $F=\mathbb{Q}$, let $c=2 s$. If $F=\mathbb{F}_{p}$, let $c=p^{s}$. If $F=\mathbb{Q}_{p}$, let $c=2 s p^{s}$.

Let $L_{0}$ be the subfield of $L$ consisting of elements algebraic over $F$. Then $\left[L: F\left(x_{1}, \ldots, x_{r}\right)\right]=s[L: K] \leq s m$, so $\left[L_{0}: F\right] \leq s m$. If $F=\mathbb{Q}$ or $F=\mathbb{F}_{p}$, it is clear that the given $c$ works. 
Suppose that $F=\mathbb{Q}_{p}$. Extend the standard absolute value on $\mathbb{Q}_{p}$ to $L_{0}$. Let $(A, \mathfrak{m}, k)$ be the valuation ring of $L_{0}$. If $x \in L_{0}$ is a root of unity, $|x|=1$, so $x \in A$. Since $k$ is an extension of $\mathbb{F}_{p}$ of degree $\leq s m$, the number of elements in $k$ is bounded by $p^{s m}$. By (7.5), it follows that for any $r \in \mathbb{N}$ which is prime to $p$ (and hence invertible in $A$ ), the number of $r$ th roots of unity in $A$ is $\leq p^{s m}$.

For $p \neq 2, \mathbb{Q}_{p}$ has no $p$ th roots of unity other than 1 (because then $p$ is unramified in $\mathbb{Q}_{p}$ but totally ramified in $\mathbb{Q}_{p}\left(\mu_{p}\right)$; cf. [23], p. 20, Exercise 14). For $p=2, \mathbb{Q}_{p}$ contains no square root of -1 . For any field $M$, let $M^{\prime}$ denote its subfield generated by $\left\{x \in M: x^{\left(p^{n}\right)}=1\right.$ for some $\left.n \in \mathbb{N}\right\}$. Then $\mathbb{Q}_{p}^{\prime}=\mathbb{Q}$. Hence $\left[L_{0}^{\prime}: \mathbb{Q}\right] \leq s m$. Hence

$$
\mid\left\{x \in L_{0}: x^{\left(p^{n}\right)}=1 \text { for some } n \in \mathbb{N}\right\} \mid \leq 2 s m .
$$

Hence the number of roots of unity in $L_{0}$ is $\leq(2 s m) p^{s m} \leq c^{m}$.

Proof of (7.4). For part (a), we may assume that $H \subset \mathrm{GL}_{r}(k)$ for some field $k$, where $n$ is invertible in $k$. Let $g_{1}, \ldots, g_{l} \in \mathrm{GL}_{r}(k)$ be distinct commuting elements with $g_{i}^{n}=1$ for each $i$. We need to prove that there is some $N \in \mathbb{N}$ (independent of $\left.g_{1}, \ldots, g_{l}\right)$ such that $l \leq N$. Let $C$ be the subalgebra of $\operatorname{Mat}_{r \times r}(k)$ generated by $g_{1}, \ldots, g_{l}$. Then $C$ is a commutative, artinian $k$-algebra, and $\operatorname{Spec}(C)$ has at most $r$ components, since $\operatorname{Mat}_{r \times r}(k)$ has at most $r$ distinct nonzero orthogonal idempotents. It follows from (7.5) that the equation $x^{n}=1$ has at most $n^{r}$ solutions in $C$. Let $N=n^{r}$.

For part (b), the field $k$ will be a finitely generated field extension of $\mathbb{Q}, \mathbb{F}_{p}$, or $\mathbb{Q}_{p}$, for some prime number $p$. Construct $C$ as in the preceding paragraph. We have to bound $l$ in terms of $r$ alone, and not in terms of $n$. We may assume that $C$ is local. The residue field $L$ of $C$ is a finite extension of $k$, and $[L: k] \leq r^{2}$. Apply (7.6) and (7.5).

We now work towards a proof of (7.1).

Lemma 7.7. Let $M$ be a finitely generated A-module. Then there exists a commutative artinian $A$-algebra $B$, such that $B$ is essentially of finite type over $A$, and such that the canonical map $M \rightarrow M \otimes_{A} B$ is injective.

Proof. Let us say that an ideal $I \subset A$ is good if there exists a commutative artinian $(A / I)$-algebra $B_{[I]}$ which is essentially of finite type over $A / I$ such that the canonical map

$$
M \otimes_{A}(A / I) \rightarrow M \otimes_{A} B_{[I]}
$$

is injective.

Let $I \subset A$ be an ideal, and suppose that every ideal properly containing $I$ is good. To prove the lemma, it suffices (by a sort of noetherian induction) to show that $I$ is good. Replacing $A$ by $A / I$, we may assume that $I=0$.

Choose a primary decomposition $0=Q_{1} \cap \cdots \cap Q_{r}$ of 0 in $M$. Then the map $M \rightarrow$ $M / Q_{1} \times \cdots \times M / Q_{r}$ is injective and each module $M / Q_{i}$ has a unique associated prime $\mathfrak{p}_{i}$.

Since each module $M / Q_{i}$ admits a filtration with quotients isomorphic to $A / \mathfrak{p}_{i}$, it follows that there exists an integer $N$ such that the maps $M / Q_{i} \rightarrow\left(M / Q_{i}\right) \otimes_{A} A / \mathfrak{p}_{i}^{N}$ are injective. Note also that the maps $M / Q_{i} \rightarrow\left(M / Q_{i}\right) \otimes_{A} A_{\mathfrak{p}_{i}}$ are injective. 
We may assume that $\mathfrak{p}_{1}, \ldots, \mathfrak{p}_{r}$ are arranged so that $\mathfrak{p}_{1}, \ldots, \mathfrak{p}_{k}$ are minimal primes of $A$ and $\mathfrak{p}_{k+1}, \ldots, \mathfrak{p}_{r}$ are not. Then $\mathfrak{p}_{k+1}^{N}, \ldots, \mathfrak{p}_{r}^{N}$ are nonzero. Let

$$
B=A_{\mathfrak{p}_{1}} \times A_{\mathfrak{p}_{k}} \times B_{\left[\mathfrak{p}_{k+1}^{N}\right]} \times B_{\left[\mathfrak{p}_{r}^{N}\right]} .
$$

We use Witt rings in this and the next section. The reader may find treatments of the subject in ([25], VIII, Exercises 42-44), ([28], II, $\S \S 5$ and 6), and [8]. Some remarks about notation are in order. There is a version of the Witt ring which does not depend on the choice of a prime number $p$, as discussed for example in ([25], VIII, Exercise 42). It seems reasonable to denote this version of the Witt ring by $W(A)$. There is a second version of the Witt ring which does depend on the choice of a prime number $p$, as discussed for example in ([25], VIII, Exercise 43). To avoid confusion, we will denote this version of the Witt ring by $W^{p}(A)$, as is done in ([8], p. 179). However, the ring we denote by $W^{p}(A)$ is the same as the ring denoted $W(A)$ in [28]. We will be using the ring $W^{p}(A)$, as well as the truncated version $W_{n}^{p}(A)$.

Lemma 7.8. Let $k$ be a perfect field of positive characteristic $p$. Fix $n \in \mathbb{N}$, and let $A=W_{n}^{p}(k)$. Let $F$ be a coherent $A$-functor. Define a $k$-functor $\widetilde{F}$ by $\widetilde{F}(B)=$ $F\left(W_{n}^{p}(B)\right)$, for all (commutative) k-algebras B. Then $\widetilde{F}$ is coherent.

Proof. We let $\sim$ denote the operation which is in effect defined in the statement. Let $r$ be the level of $F$.

Suppose that $r=0$, so we may assume that $F=\underline{M}$ for some finitely generated $A$-module $M$. Since $A=W^{p}(k) /\left(p^{n}\right)$, and $W^{p}(k)$ is a complete discrete valuation ring with uniformizing parameter $p$, it follows that $M$ may be expressed as a direct sum of modules of the form $A /\left(p^{i}\right)$, where $i \in\{1, \ldots, n\}$. We may assume that in fact $M=A /\left(p^{i}\right)$. Then

$$
\widetilde{F}(B)=A /\left(p^{i}\right) \otimes_{A} W_{n}^{p}(B)=W_{i}^{p}(B),
$$

which may be identified (as a set) with $B^{i}$. Hence $\widetilde{F}$ is coherent.

Now suppose that $r \geq 1$. By (1.1), we may choose a left exact sequence

$$
F \longmapsto G \rightarrow \underline{N}
$$

of $A$-functors, where $G$ is a coherent $A$-functor of level $r-1$ and $N$ is a finitely generated $A$-module. We obtain a left exact sequence

$$
\widetilde{F} \longmapsto \widetilde{G} \rightarrow \underline{\widetilde{N}}
$$

of $k$-functors. By induction on $r$, we may assume that $\widetilde{G}$ is coherent, and by the case $r=0, \underline{\widetilde{N}}$ is coherent. Hence $\widetilde{F}$ is coherent.

Proof of (7.1). Since $G$ is locally coherent, it is a sheaf with respect to the Zariski topology, so we may immediately reduce to the case where $X$ is affine, say $X=$ $\operatorname{Spec}(A)$. We work with $A$-functors.

By (1.1), there exist a finitely generated $A$-module $M$ and an embedding $G \hookrightarrow \underline{M}$ of $A$-functors. (Note that this map need not preserve the group structure; otherwise the proof would be much easier!) By (7.7), we can choose an artinian $A$-algebra $B$ which is essentially of finite type over $A$ such that the map $\underline{M}(A) \rightarrow \underline{M}(B)$ is injective. Let $\left.G\right|_{B}$ denote the $B$-functor given by $\left.G\right|_{B}(C)=G(C)$. Then $\left.G\right|_{B}$ is coherent by (5.4). Therefore it suffices to show that $\left.G\right|_{B}$ has the desired property. Replacing $A$ by $B$, we may reduce to the case where $A$ is artinian. Since $A$ is a 
product of Artin local rings, we may in fact reduce to the case where $A$ is an Artin local ring.

First suppose that $A$ is a field. Then $A$ is a coherent $A$-functor, so $G$ is representable by an affine group scheme of finite type over $A$. Hence $([9], 11.11) G(A)$ embeds in $\mathrm{GL}_{n}(A)$ for some $n$.

Now suppose that $A$ contains a field. Then from the Cohen structure theorem for complete local rings, we know that $A$ contains a coefficient field $k$. Since $A$ is artinian, it follows that $A$ is module-finite over $k$. By (5.5), we may reduce to the case $A=k$.

Finally, suppose that $A$ is an Artin local ring which does not contain a field. Then $A$ has mixed characteristic. Let $\mathfrak{m}$ be its maximal ideal, and let $k$ be its residue field. By ([19], 0.6.8.3), there exists a (commutative) faithfully flat noetherian local $A$-algebra $(\widetilde{A}, \tilde{\mathfrak{m}}, \tilde{k})$, with $\tilde{k}$ being an algebraic closure of $k$, such that $\mathfrak{m} \widetilde{A}=\tilde{\mathfrak{m}}$. From the latter fact, it follows that $\widetilde{A}$ is artinian. Since $G$ is coherent, by (5.1) it is a sheaf for the fpqc topology, so $F(A) \rightarrow F(\widetilde{A})$ is injective. Hence we may assume that $A=\widetilde{A}$, and so $k$ is algebraically closed. By the Cohen structure theorem, $A \cong W_{n}^{p}(k)\left[\left[x_{1}, \ldots, x_{r}\right]\right] / I$ for some $r, n \in \mathbb{N}$ and some ideal $I$.

Since $W_{n}^{p}(k)$ maps onto the residue field of $A$, and since $A$ is artinian, it follows that $A$ is module-finite over $W_{n}^{p}(k)$. By (5.5), we may reduce to the case $A=W_{n}^{p}(k)$. Apply (7.8) to reduce to the case $A=k$.

\section{Global sections - ARithmetic case}

In this section we refine the results of the last section, in the special case where $X$ is of finite type over $\mathbb{Z}$. In particular, (7.2)(b) is supplanted by (8.5).

Let us say that a group is arithmetically linear if it may be embedded as a subgroup of $\mathrm{GL}_{n}(C)$, for some $n$ and some finitely generated commutative $\mathbb{Z}$-algebra $C$. We will prove:

Theorem 8.1. Assume that $X$ is of finite type over $\mathbb{Z}$. Let $G$ be a group-valued locally coherent $X$-functor. Then $G(X)$ is arithmetically linear. Moreover, if $n$ is invertible in $\Gamma\left(X, \mathcal{O}_{X}\right)$, then the $n$-torsion in $G(X)$ is finite.

First we analyze the structure of arithmetically linear abelian groups. Recall that an abelian group $H$ is bounded if $n H=0$ for some $n \in \mathbb{N}$. It is known [see [15], 11.2, or [10], Chapter VII, §2, Exercise 12(b)] that any bounded abelian group is a direct sum of cyclic groups. Thus one may characterize the bounded abelian groups as those which can be expressed as direct sums of cyclic groups, in which the orders of the summands are bounded.

For purposes of this paper, let us say that an abelian group $H$ is cobounded if it may be embedded as a subgroup of a direct sum of (possibly infinitely many) copies of $\mathbb{Z}[1 / n]$ for some $n \in \mathbb{N}$. This is equivalent to saying that $H$ is torsion-free and that $H \otimes_{\mathbb{Z}} \mathbb{Z}[1 / n]$ is a free $\mathbb{Z}[1 / n]$-module for some $n$.

Remark 8.2. We do not know of a structure theorem for abelian groups which are countable and cobounded. Certainly such groups can be rather complicated. For example, not every subgroup $H$ of $\bigoplus_{k=1}^{\infty} \mathbb{Z}[1 / 2]$ can be expressed as a direct sum of copies of $\mathbb{Z}$ and copies of $\mathbb{Z}[1 / 2]$; consider:

$$
H=\left\{a \in \bigoplus_{k=1}^{\infty} \mathbb{Z}[1 / 2]: \sum_{k=1}^{\infty} \frac{a_{k}}{k} \in \mathbb{Z}\right\} .
$$


Let $L$ be the maximal 2-divisible subgroup of $H$. Then $H / L \cong \mathbb{Q}$.

Let us say that an abelian group $H$ is bounded $\times$ cobounded if $H \cong B \times C$ for some bounded group $B$ and some cobounded group $C$. We shall see (8.5) that arithmetically linear abelian groups are the same as countable (bounded $\times$ cobounded) abelian groups.

Lemma 8.3. (a) Let $M$ be a bounded $\times$ cobounded abelian group, and let $H$ be a subgroup of $M$. Then $H$ is bounded $\times$ cobounded.

(b) Let

$$
0 \rightarrow M^{\prime} \rightarrow M \rightarrow M^{\prime \prime} \rightarrow 0
$$

be a short exact sequence of abelian groups, in which $M^{\prime}$ and $M^{\prime \prime}$ are bounded $\times$ cobounded. Then $M$ is bounded $\times$ cobounded.

Proof. Part (a) follows from ([15], 50.3): if the torsion subgroup of an abelian group is bounded, then it is a direct summand.

For part (b), write $M^{\prime}=B^{\prime} \times C^{\prime}$ and $M^{\prime \prime}=B^{\prime \prime} \times C^{\prime \prime}$ where $B^{\prime}, B^{\prime \prime}$ are bounded and $C^{\prime}, C^{\prime \prime}$ are cobounded. Let $M_{\text {tor }}$ be the torsion subgroup of $M$. We have a left exact sequence:

$$
0 \rightarrow B^{\prime} \rightarrow M_{\text {tor }} \rightarrow B^{\prime \prime},
$$

from which it follows that $M_{\text {tor }}$ is bounded, and hence that $M_{\text {tor }}$ is a direct summand of $M$. Therefore it suffices to show that $M / M_{\text {tor }}$ is cobounded. We have an exact sequence:

$$
0 \rightarrow C^{\prime} \rightarrow M / M_{\text {tor }} \rightarrow \overline{B^{\prime \prime}} \times C^{\prime \prime} \rightarrow 0,
$$

in which $\overline{B^{\prime \prime}}$ is a quotient of $B^{\prime \prime}$ and hence is bounded. Choose $n \in \mathbb{N}$ such that $n \overline{B^{\prime \prime}}=0$, and such that $n$ satisfies the property of $n$ in the definition of cobounded, for both $C^{\prime}$ and $C^{\prime \prime}$. Tensoring by $\mathbb{Z}[1 / n]$ yields an exact sequence:

$$
0 \rightarrow C^{\prime}[1 / n] \rightarrow\left(M / M_{\text {tor }}\right)[1 / n] \rightarrow C^{\prime \prime}[1 / n] \rightarrow 0 .
$$

By construction, $C^{\prime}[1 / n]$ and $C^{\prime \prime}[1 / n]$ are submodules of free $\mathbb{Z}[1 / n]$-modules and hence are themselves free. Hence $\left(M / M_{\text {tor }}\right)[1 / n]$ is free, so $M / M_{\text {tor }}$ is cobounded.

Lemma 8.4. Let $A$ be a commutative $\mathbb{Z}$-algebra of finite type. Let $M$ be a finitely generated $A$-module. Then the abelian group $M$ is bounded $\times$ cobounded.

Proof. As an abelian group, we may embed $M$ as a subgroup of the additive group of the symmetric algebra of $M$, which is a finitely generated $A$-algebra. Therefore we may reduce to the case $M=A$.

Take a primary decomposition $0=\mathfrak{q}_{1} \cap \cdots \cap \mathfrak{q}_{m}$ of 0 in $A$. Then the canonical map $A \rightarrow \prod_{i=1}^{n} A / \mathfrak{q}_{i}$ is injective, so we may reduce to the case where $A$ has a unique associated prime.

Let $q$ be the characteristic of $A$. First suppose that $q>0$. Then $q A=0$, so $A$ is bounded.

Now suppose that $q=0$. By the Noether normalization lemma, there exist elements $x_{1}, \ldots, x_{r} \in A \otimes_{\mathbb{Z}} \mathbb{Q}$ which are algebraically independent over $\mathbb{Q}$ and such that $A \otimes_{\mathbb{Z}} \mathbb{Q}$ is module-finite over $\mathbb{Q}\left[x_{1}, \ldots, x_{r}\right]$. We may assume that $x_{1}, \ldots, x_{r} \in A$. Let $S=\operatorname{Spec}(\mathbb{Z}), X=\operatorname{Spec}(A), Y=\operatorname{Spec}\left(\mathbb{Z}\left[x_{1}, \ldots, x_{r}\right]\right)$, so we have a morphism $\phi: X \rightarrow Y$ of $S$-schemes. If $\eta \in S$ is the generic point, then $\phi_{\eta}$ is finite, so it follows from ([18], 8.1.2(a), 8.10.5(xii), 8.11.1, 9.6.1(vii)) that for some 
$n \in \mathbb{N}, \phi \otimes_{S} \operatorname{Spec}(\mathbb{Z}[1 / n])$ is finite, i.e. that $A_{n}=A \otimes_{\mathbb{Z}} \mathbb{Z}[1 / n]$ is a module-finite $\mathbb{Z}\left[1 / n, x_{1}, \ldots, x_{r}\right]$-algebra. Since $A$ has characteristic zero and has a unique associated prime, it follows that $A_{n}$ is a torsion-free $\mathbb{Z}\left[1 / n, x_{1}, \ldots, x_{r}\right]$-module and that the canonical map $A \rightarrow A_{n}$ is injective. Hence $A$ embeds (as an abelian group) in $\left(\mathbb{Z}\left[1 / n, x_{1}, \ldots, x_{r}\right]\right)^{k}$ for some $k$, so $A$ is cobounded.

Proposition 8.5. Let $H$ be an abelian group. Then $H$ is arithmetically linear if and only if it is countable and bounded $\times$ cobounded.

Proof. First suppose that $H$ is countable and bounded $\times$ cobounded. For some $n, k \in \mathbb{N}$ and some prime numbers $p_{1}, \ldots, p_{k}$, we may embed $H$ as a subgroup of a countable direct sum $K$ of copies of

$$
\mathbb{Z}[1 / n] \oplus\left(\bigoplus_{i=1}^{k} \mathbb{Z} / p_{i}^{n} \mathbb{Z}\right) .
$$

Then $K$ is the additive group of the ring

$$
A=\mathbb{Z}[1 / n, t] \times\left(\mathbb{Z} / p_{1}^{n} \mathbb{Z}\right)[t] \times \cdots \times\left(\mathbb{Z} / p_{k}^{n} \mathbb{Z}\right)[t],
$$

so $K$ may be embedded as a subgroup of $\mathrm{GL}_{2}(A)$. Hence $H$ is arithmetically linear.

Now suppose that $H$ is arithmetically linear. The countability of $H$ is clear. Embed $H$ as a subgroup of $\mathrm{GL}_{r}(A)$, for some finitely generated commutative $\mathbb{Z}$ algebra $A$. Let $R$ be the sub- $A$-algebra of $\operatorname{Mat}_{r \times r}(A)$ generated by $H$. Then $R$ is a finite $A$-algebra, and $H$ is a subgroup of $R^{*}$. By (8.3)(a), it suffices to show that $R^{*}$ is bounded $\times$ cobounded. We may as well view $R$ as an arbitrary finitely generated commutative $\mathbb{Z}$-algebra.

Let $J$ be the nilradical of $R$. For each $n \in \mathbb{N}$, there is a short exact sequence of abelian groups

$$
0 \rightarrow J^{n} / J^{n+1} \rightarrow\left(R / J^{n+1}\right)^{*} \rightarrow\left(R / J^{n}\right)^{*} \rightarrow 1 .
$$

The group $(R / J)^{*}$ is finitely generated (see $(9.9)$ ), and hence is bounded $\times$ cobounded. Hence by $(8.3)(\mathrm{b})$, it suffices to show that $J^{n} / J^{n+1}$ is bounded $\times$ cobounded. Apply (8.4).

We now work towards a proof of (8.1).

Lemma 8.6. Fix $n \in \mathbb{N}$ and a prime number $p$. Let $S \subset W_{n}^{p}(A)$ be a multiplicatively closed set. Let $\mu: W_{n}^{p}(A) \rightarrow A$ be the canonical map. Let $\bar{S}=\mu(S)$. Then the canonical map $i: W_{n}^{p}(A) \rightarrow W_{n}^{p}\left(\bar{S}^{-1} A\right)$ factors through $S^{-1} W_{n}^{p}(A)$.

Proof. Let $\nu: W_{n}^{p}\left(\bar{S}^{-1} A\right) \rightarrow \bar{S}^{-1} A$ be the canonical map. Let $f \in S$. Then the image of $\mu(f)$ in $\bar{S}^{-1} A$ is invertible, so $\nu(i(f))$ is invertible. But $\nu$ is surjective and has nilpotent kernel, so $i(f)$ is invertible.

Note that if $A$ is any $\mathbb{F}_{p}$-algebra, there is a canonical map $W_{n}^{p}\left(\mathbb{F}_{p}\right) \rightarrow W_{n}^{p}(A)$, and since $W_{n}^{p}\left(\mathbb{F}_{p}\right)=\mathbb{Z} / p^{n} \mathbb{Z}$, we see that $p^{n}=0$ in $W_{n}^{p}(A)$.

Proposition 8.7. For any $n, k \in \mathbb{N}$, and any prime number $p$, the ring

$$
W_{n}^{p}\left(\mathbb{F}_{p}\left[t_{1}, \ldots, t_{k}\right]\right)
$$

is isomorphic to the subring of $\left(\mathbb{Z} / p^{n} \mathbb{Z}\right)\left[t_{1}^{1 / p^{n-1}}, \ldots, t_{k}^{1 / p^{n-1}}\right]$ generated by the elements $p^{r} t_{i}^{j / p^{r}}$ for $0 \leq r \leq n-1,1 \leq i \leq k$, and $1 \leq j \leq p-1$. 
Proof. Let $R=\mathbb{F}_{p}\left[t_{1}^{p^{-\infty}}, \ldots, t_{k}^{p^{-\infty}}\right]$, and let $V=W_{n}^{p}(R)$. Let

$$
W=\left(\mathbb{Z} / p^{n} \mathbb{Z}\right)\left[t_{1}^{p^{-\infty}}, \ldots, t_{k}^{p^{-\infty}}\right] .
$$

It is easily seen that there exists a unique ring homomorphism $\eta: W \rightarrow V$ with the property that $\eta\left(t_{i}^{p^{-m}}\right)=\left(t_{i}^{p^{-m}}, 0, \ldots, 0\right)$ for each $i$ and each $m \geq 0$.

We show that $\eta$ is surjective. Let $\mu: V \rightarrow R$ be the canonical map. Since $R$ is perfect, $\operatorname{Ker}(\mu)=(p)$. Because of this, because $p^{n}=0$ in $V$, and because $t_{i}^{1 / p^{r}}$ (for various $i, r)$ generate $R$ as a $\mathbb{Z}$-algebra, it follows that the $\eta\left(t_{i}^{1 / p^{r}}\right)$ generate $V$ as a $\mathbb{Z}$-algebra. Hence $\eta$ is surjective.

We show that $\eta$ is injective. Suppose otherwise. Let $x \in \operatorname{Ker}(\eta), x \neq 0$. We may assume that $p x=0$. Then $x=p^{n-1} y$ for some $y \in W$; we may assume that the coefficients which appear in $y$ lie in the set $\{1, \ldots, p-1\}$. It follows that the 0 th component of $\eta(y)$ is nonzero. Hence $p^{n-1} \eta(y) \neq 0$, so $\eta(x) \neq 0$; contradiction. Hence $\eta$ is injective.

We return to the proof of the proposition. Let $A_{n}=W_{n}^{p}\left(\mathbb{F}_{p}\left[t_{1}, \ldots, t_{k}\right]\right)$. From what we have just done, it follows that $A_{n}$ may be identified with a subring of $W$. Since $\eta\left(p^{r} t_{i}^{j / p^{r}}\right)=\left(0, \ldots, 0, t_{i}^{j}, 0, \ldots, 0\right)$, where $t_{i}^{j}$ appears in the $r$ th spot, it follows that $p^{r} t_{i}^{j / p^{r}} \in A_{n}$, for each $i, r$, and $j$. To complete the proof, we must show that these elements generate $A_{n}$. Let $A_{n}^{\prime}$ be the subring of $A_{n}$ generated by the elements $p^{r} t_{i}^{j / p^{r}}$. Consider the canonical map $\tau: A_{n} \rightarrow A_{n-1}$. By induction on $n$ we may assume that the elements $p^{r} t_{i}^{j / p^{r}}$ generate $A_{n-1}$, and so that $\tau\left(A_{n}^{\prime}\right)=A_{n-1}$. Let $f \in \mathbb{F}_{p}\left[t_{1}, \ldots, t_{k}\right]$. Write $f=\sum_{I} a_{I} t^{I}$, where $I$ is a multiindex. Since $a_{I} \in\{0, \ldots, p-1\}$, we may view $a_{I}$ as an element of $\mathbb{Z} / p^{n} \mathbb{Z}$. Let $\tilde{f}=p^{n-1} \sum_{I} a_{I}\left(t^{I}\right)^{1 / p^{n-1}} \in W$. Then $\tilde{f}$ corresponds to the element $(0, \ldots, 0, f)$ of $A_{n}$. Hence $\operatorname{Ker}(\tau) \subset A_{n}^{\prime}$. Since $\tau\left(A_{n}^{\prime}\right)=A_{n-1}$, it follows that $A_{n}^{\prime}=A_{n}$.

Corollary 8.8. Fix $n \in \mathbb{N}$ and a prime number $p$. Let $A$ be an $\mathbb{F}_{p}$-algebra of finite type. Then $W_{n}^{p}(A)$ is a $\mathbb{Z}$-algebra of finite type.

Proof. Choose a surjection $\pi: \mathbb{F}_{p}\left[x_{1}, \ldots, x_{k}\right] \rightarrow A$. Then $W_{n}^{p}(\pi)$ is a surjection, and since (8.7) $W_{n}^{p}\left(\mathbb{F}_{p}\left[x_{1}, \ldots, x_{k}\right]\right)$ is of finite type over $\mathbb{Z}$, so is $W_{n}^{p}(A)$.

The following result is a variant of (7.8).

Lemma 8.9. Fix $n \in \mathbb{N}$ and a prime number $p$. Assume that $A$ is an $\mathbb{F}_{p}$-algebra of finite type. Let $C=W_{n}^{p}(A)$. (By (8.8), $C$ is noetherian.) Let $F$ be a coherent $C$-functor, built up from $\left\{C /\left(p^{k}\right)\right\}_{1 \leq k \leq n}$. Let $G$ be the A-functor given by $G(B)=$ $F\left(W_{n}^{p}(B)\right)$. Let $\widetilde{G}$ be the sheaf associated to $G$ for the ffqc topology. Then $\widetilde{G}$ is representable.

Remarks. The functor $G$ is not in general coherent, as it is not in general an ffqc sheaf. For an arbitrary coherent $C$-functor $F$, with no restrictions on how it is built up, it may be that the corresponding functor $\widetilde{G}$ is always representable.

Proof of (8.9). Since any limit of finitely many representable functors is representable, we may assume that $F=\underline{C /\left(p^{k}\right)}$ for some $k$. Then we may describe $G$ by

$$
G(B)=\frac{\left\{\left(b_{0}, \ldots, b_{n-1}\right): b_{i} \in B \text { for all } i\right\}}{\left\{\left(0, \ldots, 0, d_{k}^{p^{k}}, \ldots, d_{n-1}^{p^{k}}\right): d_{i} \in B \text { for all } i\right\}},
$$


where it is to be understood that this quotient of abelian groups takes place with respect to the abelian group structure on $W_{n}^{p}(B)$. For any $b \in B$, there exists a faithfully flat ring extension $\phi: B \rightarrow B^{\prime}$ such that $\phi(b)$ is a $\left(p^{k}\right)$ th power. Therefore to complete the proof, it suffices to show that the $A$-functor $H$ given by

$$
H(B)=\frac{\left\{\left(b_{0}, \ldots, b_{n-1}\right): b_{i} \in B \text { for all } i\right\}}{\left\{\left(0, \ldots, 0, e_{k}, \ldots, e_{n-1}\right): e_{i} \in B \text { for all } i\right\}}
$$

is representable. (Then we will have $H=\widetilde{G}$.) But

$$
\begin{aligned}
H(B) & \cong W_{n}^{p}(B) / \operatorname{Ker}\left[W_{n}^{p}(B) \rightarrow W_{k}^{p}(B)\right] \\
& \cong W_{k}^{p}(B) \cong B^{k},
\end{aligned}
$$

so $H$ is representable.

Let $A=\mathbb{F}_{p}\left[t_{1}, \ldots, t_{k}\right]$. Let $C=\left(\mathbb{Z} / p^{n} \mathbb{Z}\right)\left[t_{1}, \ldots, t_{k}\right]$. There is a canonical map $\phi: C \rightarrow W_{n}^{p}(A)$ given by setting $\phi\left(t_{i}\right)=\left(t_{i}, 0, \ldots, 0\right)$ for each $i$.

Proposition 8.10. Let $A=\mathbb{F}_{p}\left[t_{1}, \ldots, t_{k}\right]$. Let $C=\left(\mathbb{Z} / p^{n} \mathbb{Z}\right)\left[t_{1}, \ldots, t_{k}\right]$. Let $S$ be a multiplicatively closed subset of $C$. Let $\pi: C \rightarrow A$ be the canonical map. Let $F$ be a coherent $S^{-1} C$-functor. Assume that $F$ is built up from $\left\{S^{-1} C /\left(p^{r}\right)\right\}_{1 \leq r \leq n}$. Let $\bar{S}=\pi(S)$. Let $G$ be the ffqc sheaf associated to the $\bar{S}^{-1} A$-functor given by $B \mapsto F\left(W_{n}^{p}(B)\right)$. (This makes sense by (8.6).) Then:

(a) $G$ is representable;

(b) the canonical map $i: F\left(S^{-1} C\right) \rightarrow G\left(\bar{S}^{-1} A\right)$ is injective.

Proof. (a) Let $D=W_{n}^{p}\left(\bar{S}^{-1} A\right)$. Let $\phi: \operatorname{Spec}(D) \rightarrow \operatorname{Spec}\left(S^{-1} C\right)$ be the canonical map. Then $\phi^{*} F$ is a $D$-functor, which is coherent (see (5.4)), and is in fact built up from $\left\{D /\left(p^{r}\right)\right\}_{1 \leq r \leq n}$. Apply (8.9).

(b) The construction is functorial in $F$, so we may reduce to the case where $F=\underline{S^{-1} C /\left(p^{r}\right)}$. In that case, one sees that $i$ is isomorphic to the canonical map

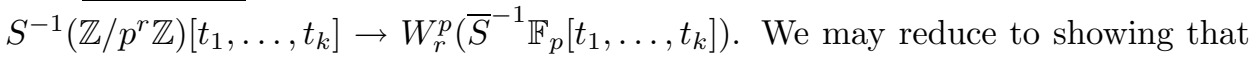
the canonical map $j:\left(\mathbb{Z} / p^{r} \mathbb{Z}\right)\left[t_{1}, \ldots, t_{k}\right] \rightarrow W_{r}^{p}\left(\mathbb{F}_{p}\left[t_{1}, \ldots, t_{k}\right]\right)$ is injective. This follows from the proof of (8.7).

Lemma 8.11. Assume that $A$ is a domain. Let $F$ be a coherent A-functor. Then for some $f \in A-\{0\}$, the pullback [along $\left.\operatorname{Spec}\left(A_{f}\right) \rightarrow \operatorname{Spec}(A)\right]$ of $F$ to $A_{f}$ is representable by an A-algebra of finite type.

Proof (Sketch). The functor $F$ is built up from finitely many $A$-modules, each finitely generated. Pick some $f \in A-\{0\}$ such that the localization of each such module at $f$ is free.

Lemma 8.12. Assume that $X$ is integral. Let $G$ be an affine group scheme of finite type over $X$. Then there exist some $n \in \mathbb{N}$, a nonempty open subscheme $U \subset X$, and a closed immersion $G_{U} \rightarrow \mathrm{GL}_{n}(U)$ of $U$-schemes which is also a homomorphism.

Proof (Sketch). Let $\eta$ be the generic point of $X$. For some $n \in \mathbb{N}$, we have a closed immersion (and a homomorphism) $h: G_{\eta} \rightarrow \operatorname{GL}_{n}(X)_{\eta}$ of Spec $k(\eta)$-schemes. There is a nonempty open subscheme $V \subset X$ and a closed immersion $G_{V} \rightarrow \mathrm{GL}_{n}(V)$ of $V$-schemes which induces $h$. Replacing $V$ by a sufficiently small nonempty open subscheme $U$, we obtain (by restriction) a homomorphism (and a closed immersion) $G_{U} \rightarrow \mathrm{GL}_{n}(U)$ of $U$-schemes. 
Lemma 8.13. Let $A=\left(\mathbb{Z} / p^{n} \mathbb{Z}\right)\left[t_{1}, \ldots, t_{k}\right]$. Let $M$ be a finitely generated $A$ module. Then there exist a non-zero-divisor $f \in A$ and positive integers $l_{1}, \ldots, l_{r}$ such that $M_{f} \cong A_{f} /\left(p^{l_{1}}\right) \oplus \cdots \oplus A_{f} /\left(p^{l_{r}}\right)$ as $A_{f}$-modules.

Proof (Sketch). Let $S$ be the set of non-zero-divisors of $A$. In $S^{-1} A$, the only elements (up to associates) are $1, p, \ldots, p^{n-1}, 0$. Hence every ideal of $S^{-1} A$ is principal, so every finitely generated $S^{-1} A$ module is a direct sum of cyclic modules, necessarily of the form $S^{-1} A /\left(p^{j}\right)$ for various $j$. The lemma follows.

Proof of (8.1). The comment about what happens when $n$ is invertible in $\Gamma\left(X, \mathcal{O}_{X}\right)$ follows from (7.2)(b), so a direct proof is omitted.

Some of the steps here follow the proof of (7.1). We may assume that $X$ is affine, $X=\operatorname{Spec}(A)$. Choose $M$ and $B$ as in the proof of (7.1). Write $B=S^{-1} C$ for some finitely generated $A$-algebra $C$ and some multiplicatively closed subset $S \subset C$. Certainly we may replace $A$ by $C$, so $B=S^{-1} A$. Replacing $A$ by $A_{g}$ for some suitably chosen $g \in S$, we may assume that the connected components of $\operatorname{Spec}(A)$ are irreducible and that they correspond bijectively with the points of $\operatorname{Spec}\left(S^{-1} A\right)$. Write $A=A_{1} \times \cdots \times A_{m}$, where $\operatorname{Spec}\left(A_{i}\right)$ is irreducible for each $i$. Localizing further if necessary, we may assume that $A_{i}$ has a unique associated prime for each $i$.

We may reduce to the situation where $A$ has a unique associated prime and $S^{-1} A$ is an Artin local ring. It suffices to show that for some $f \in S, G\left(A_{f}\right)$ is arithmetically linear. Let $r$ be the characteristic of $A$.

Since $A$ has a unique associated prime and $S^{-1} A$ is an Artin local ring, every nonnilpotent element of $A$ lies in $S$.

First suppose that $r=0$. By Noether normalization, we may find algebraically independent elements $x_{1}, \ldots, x_{s} \in A$ such that $A \otimes_{\mathbb{Z}} \mathbb{Q}$ is module-finite over $\mathbb{Q}\left[x_{1}, \ldots, x_{s}\right]$. As in the proof of $(8.4)$, there is some $n \in \mathbb{N}$ such that $A \otimes_{\mathbb{Z}} \mathbb{Z}[1 / n]$ is a module-finite $\mathbb{Z}\left[1 / n, x_{1}, \ldots, x_{s}\right]$-algebra. Every nonzero element of $\mathbb{Z}\left[x_{1}, \ldots, x_{s}\right]$ lies in $S$. We may replace $A$ by $A \otimes_{\mathbb{Z}} \mathbb{Z}[1 / n]$. By (5.5)(b), the pushforward of $G$ to $\mathbb{Z}\left[1 / n, x_{1}, \ldots, x_{s}\right]$ is coherent. By $(8.11)$, there is some $f \in \mathbb{Z}\left[1 / n, x_{1}, \ldots, x_{s}\right]-\{0\}$ such that the pullback of $G$ to $\mathbb{Z}\left[1 / n, x_{1}, \ldots, x_{s}, f^{-1}\right]$ is representable by an affine $\mathbb{Z}\left[1 / n, x_{1}, \ldots, x_{s}, f^{-1}\right]$-algebra of finite type. Apply (8.12).

Now suppose that $r>0$. Then $r=p^{m}$ for some prime $p$ and some $m$. By the Noether normalization theorem, we may find algebraically independent elements $x_{1}, \ldots, x_{s} \in A$ such that $A \otimes_{\mathbb{Z}} \mathbb{F}_{p}$ is module-finite over $\mathbb{F}_{p}\left[x_{1}, \ldots, x_{s}\right]$. It follows that $A$ is module-finite over $E=\left(\mathbb{Z} / p^{m} \mathbb{Z}\right)\left[x_{1}, \ldots, x_{s}\right]$. By $(5.5)(\mathrm{b})$, the pushforward $H$ of $G$ to $E$ is coherent, so we may reduce to the case where $A=\left(\mathbb{Z} / p^{m} \mathbb{Z}\right)\left[x_{1}, \ldots, x_{s}\right]$. Let $M_{1}, \ldots, M_{d}$ be $A$-modules from which $G$ can be built up. By (8.13), there is some $f \in S$ such that each $\left(M_{i}\right)_{f}$ is a direct sum of modules of the form $A_{f} /\left(p^{r}\right)$, for various $r$. Let $G_{f}$ be the pullback of $G$ to $A_{f}$. Then $G_{f}$ is built up from $\left\{A_{f} /\left(p^{r}\right)\right\}_{1 \leq r \leq n}$. The theory follows now by applying (8.10) and (8.12).

Problem 8.14. Which arithmetically linear groups arise as $G(X)$, for some scheme $X$ of finite type over $\mathbb{Z}$ and some group-valued coherent $X$-functor $G$ ?

Certain groups can be shown to be quotients of arithmetically linear abelian groups by finitely generated subgroups. For example, in the next section we shall see that this is the case for $\operatorname{Pic}(X)$, where $X$ is a reduced scheme of finite type over $\mathbb{Z}$. Although it may in fact be the case that $\operatorname{Pic}(X)$ is itself arithmetically linear, we have not been able to show this, so we are led to the following lemma: 
Lemma 8.15. Let $G$ be an arithmetically linear abelian group. Let $H$ be a finitely generated subgroup of $G$. Then the torsion subgroup of $G / H$ is supported at a finite set of primes.

Proof. Write $G=B \times C$, where $B$ is bounded and $C$ is cobounded. For any abelian group $M$, let $M[1 / n]$ denote $M \otimes_{\mathbb{Z}} \mathbb{Z}[1 / n]$. Choose $n \in \mathbb{N}$ such that $n B=0$ and such that $C[1 / n]$ is a free $\mathbb{Z}[1 / n]$-module. Let $Q=G / H$. It suffices to show that the torsion subgroup of $Q[1 / n]$ is supported at a finite set of primes. We have an exact sequence

$$
0 \rightarrow H[1 / n] \rightarrow C[1 / n] \rightarrow Q[1 / n] \rightarrow 0
$$

of $\mathbb{Z}[1 / n]$-modules. Since $H[1 / n]$ is contained in a finitely generated direct summand of $C[1 / n]$, it suffices to show that for any finitely generated $\mathbb{Z}[1 / n]$-module $M$, the torsion subgroup of $M$ is supported at a finite set of primes. This is easily checked.

\section{Application to the Picard group}

Let $M$ be a finitely generated $A$-module. Let $\underline{\mathrm{PGL}}(M)$ denote the Zariski sheaf associated to the $A$-functor given by

$$
B \mapsto \frac{\operatorname{Aut}_{B}\left(M \otimes_{A} B\right)}{B^{*}} .
$$

More generally, let $\mathcal{M}$ be a coherent $\mathcal{O}_{X}$-module. Let $\underline{\mathrm{PGL}}(\mathcal{M})$ denote the Zariski sheaf associated to the $X$-functor given by

$$
Y \mapsto \frac{\operatorname{Aut}_{Y}\left(\mathcal{M}_{Y}\right)}{\Gamma\left(\mathcal{O}_{Y}^{*}\right)}
$$

Since $\underline{\operatorname{Aut}}(\mathcal{M})$ acts by conjugation on $\underline{\operatorname{End}}(\mathcal{M})$, we obtain a canonical morphism of group-valued $X$-functors:

$$
\psi: \underline{\operatorname{PGL}}(\mathcal{M}) \rightarrow \text { Aut }_{\text {alg }}(\underline{\operatorname{End}}(\mathcal{M}))^{2}
$$

The $X$-functor $\mathbf{A u t}_{\text {alg }}(\operatorname{End}(\mathcal{M}))$ is locally coherent by (4.13) and example (6) from $\S 4$; it is coherent, at least assuming that $X$ is separated, by (5.11).

We would like to show that $\underline{\operatorname{PGL}}(\mathcal{M})$ is coherent. We could do this by showing that $\psi$ is an isomorphism. However, except for the case where $\mathcal{M}$ is locally free, we do not know if $\psi$ is an isomorphism. Therefore, we settle for showing that (under certain special circumstances) $\psi(X)$ is injective. This is a weak substitute for showing that $\mathrm{PGL}(\mathcal{M})$ is coherent. ${ }^{3}$

First we prove a lemma, then a corollary which says something directly about $\psi$.

Lemma 9.1. Assume that $A$ is reduced. Let $A^{\prime}$ be a ring, with $A \subset A^{\prime} \subset A_{\text {nor }}$. Assume that the ideal $\left[A: A^{\prime}\right]$ of $A$ is prime. Let $\sigma$ be an endomorphism of $A^{\prime}$ as an $A$-module. Assume that for every $\mathfrak{p} \in \operatorname{Spec}(A), \sigma \otimes_{A} k(\mathfrak{p})$ is a homothety of $A^{\prime} \otimes_{A} k(\mathfrak{p})$ as a $k(\mathfrak{p})$-module. Then $\sigma$ is a homothety of $A^{\prime}$ as an $A$-module.

\footnotetext{
${ }^{2}$ For the actual arguments which we use, one could substitute the simpler functor $\operatorname{Aut}(\underline{\operatorname{End}}(\mathcal{M}))$, but we use $\mathbf{A u t}_{\text {alg }}(\underline{\operatorname{End}}(\mathcal{M}))$ instead for aesthetic reasons because it makes $\psi$ closer to an isomorphism.

${ }^{3}$ In trying to show that $\psi$ is a monomorphism, one comes to the following question: Let $M$ be a finitely generated $A$-module. Let $\sigma$ be an automorphism of $M$ as an $A$-module. Assume that for every commutative $A$-algebra $B, \sigma \otimes_{A} B$ lies in the center of $\operatorname{End}_{B}\left(M \otimes_{A} B\right)$. Does it follow that $\sigma$ is a homothety, i.e. that $\sigma$ is given by multiplication by an element of $A$ ?
} 
Proof. First we show that $\sigma$ is given by multiplication by $\sigma(1)$. By subtracting the endomorphism of $A^{\prime}$ given by multiplication by $\sigma(1)$, we may reduce to showing that if $\sigma(1)=0$, then $\sigma=0$. Let $\mathfrak{p}_{1}, \ldots, \mathfrak{p}_{r}$ be the minimal primes of $A$. Using the fact that $\sigma \otimes_{A} k\left(\mathfrak{p}_{i}\right)$ is a homothety, we conclude that $\sigma \otimes_{A} k\left(\mathfrak{p}_{i}\right)=0$. Let $x \in A^{\prime}$. Then $\sigma(x) \mapsto 0$ in $A^{\prime} \otimes_{A} k\left(\mathfrak{p}_{i}\right)$. Since the map

$$
A^{\prime} \rightarrow \bigoplus_{i=1}^{r} A^{\prime} \otimes_{A} k\left(\mathfrak{p}_{i}\right)
$$

is injective, it follows that $\sigma(x)=0$, and hence that $\sigma=0$. Hence (reverting to the original problem), we see that $\sigma$ is given by multiplication by $\sigma(1)$.

Let $Q=A^{\prime} / A$, which is an $A$-module. Let $\mathfrak{p}=\left[A: A^{\prime}\right]$. Let $\overline{\sigma(1)}$ denote the image of $\sigma(1)$ in $Q$. It follows that $\overline{\sigma(1)} \mapsto 0$ in $Q \otimes_{A} k(\mathfrak{p})$. But by the construction of $\mathfrak{p}$, the canonical map $Q \rightarrow Q \otimes_{A} k(\mathfrak{p})$ is injective. Hence $\overline{\sigma(1)}=0$. Hence $\sigma(1) \in A$.

Corollary 9.2. Assume that $A$ is reduced. Let $A^{\prime}$ be a ring, with $A \subset A^{\prime} \subset A_{\text {nor }}$. Assume that the ideal $\left[A: A^{\prime}\right]$ of $A$ is prime. Assume that $A^{\prime}$ is a finitely generated A-module. Let

$$
\psi: \underline{\operatorname{PGL}}\left(A^{\prime}\right) \rightarrow \operatorname{Aut}_{\mathrm{alg}}\left(\underline{\operatorname{End}}\left(A^{\prime}\right)\right)
$$

be the canonical (conjugation) morphism of group-valued A-functors. Then $\psi(A)$ is injective.

Proof. Consider the morphism $\psi_{0}: \underline{\operatorname{Aut}}\left(A^{\prime}\right) \rightarrow \operatorname{Aut}_{\text {alg }}\left(\underline{\operatorname{End}}\left(A^{\prime}\right)\right)$ which induces $\psi$. Then $\operatorname{ker}\left(\psi_{0}(A)\right)$ consists of those automorphisms $\sigma$ of $A^{\prime}$ as an $A$-module with the property that $\sigma \otimes_{A} B \in \mathbb{Z}\left[\operatorname{End}_{B}\left(A^{\prime} \otimes_{A} B\right)\right]$ for every commutative $A$-algebra $B$. If $B$ is a field, it follows that for such a $\sigma, \sigma \otimes_{A} B$ is given by multiplication by an element of $B$. By (9.1), such a $\sigma$ is itself a homothety. Hence $\operatorname{ker}\left(\psi_{0}(A)\right)=A^{*}$.

Let $f \in A-\{0\}$. Then the ideal $\left[A_{f}: A_{f}^{\prime}\right]$ of $A_{f}$ is prime, and it follows that $\operatorname{ker}\left(\psi_{0}\left(A_{f}\right)\right)=A_{f}^{*}$. Hence the map

$$
\frac{\operatorname{Aut}_{A_{f}}\left(A^{\prime} \otimes_{A} A_{f}\right)}{A_{f}^{*}} \rightarrow \mathbf{A u t}_{\mathrm{alg}}\left(\underline{\operatorname{End}}\left(A^{\prime}\right)\right)\left(A_{f}\right)
$$

is injective. By considering the sheafification which occurs in the definition of $\underline{\operatorname{PGL}}\left(A^{\prime}\right)$, we see that $\psi(A)$ is injective.

Lemma 9.3. Let $C$ be an overring of $A$, with $C$ finitely generated as an $A$-module. Then there exists a chain

$$
A=A_{0} \subset A_{1} \subset \cdots \subset A_{n}=C
$$

of rings such that for each $k=1, \ldots, n$, the ideal $\left[A_{k-1}: A_{k}\right]$ of $A_{k-1}$ is prime.

Proof. We may assume that $C \neq A$. For each $y \in C-A$, let $I_{y}=[A: A[y]]$. (All conductors are to be computed as ideals in $A$.) Choose $y \in C-A$ so that $I_{y}$ is maximal amongst all such ideals. We will show that $I_{y}$ is prime. This will complete the proof.

Pick $a \in A$ such that $\left[I_{y}: a\right]$ is prime. Choose $n$ so that $y$ satisfies a monic polynomial of degree $n$ with coefficients in $A$. Then $I_{y}=\left[A: y, y^{2}, \ldots, y^{n-1}\right]$, and $\left[I_{y}: a\right]=\left[A: a y, a y^{2}, \ldots, a y^{n-1}\right]$.

First we show that $a y \notin A$. Suppose otherwise. Since $\left[I_{y}: a\right] \neq A, a \notin I_{y}$. Hence $a y^{k} \notin A$, for some $k$ with $2 \leq k \leq n-1$. Consider all pairs $(r, s) \in \mathbb{N}^{2}, s<n$, such 
that $a^{r} y^{s} \notin A$. Choose such a pair $\left(r_{0}, s_{0}\right)$ such that the ratio $s_{0} / r_{0}$ is as small as possible. (This is possible because $a y \in A$, and hence $a^{r} y^{s} \in A$ for all $r \geq s$.)

Let $(r, s) \in \mathbb{N}^{2}$ be such that $a^{r} y^{s} \notin A$. We will show that $s_{0} / r_{0} \leq s / r$. Suppose otherwise: $s_{0} / r_{0}>s / r$. We may assume that $s \geq n$. We have

$$
a^{r} y^{s}=\prod_{i=0}^{n-1} c_{i} a^{r} y^{i}
$$

for suitable $c_{i} \in A$. For $i$ in the given range we have $s / r>i / r$, so $s_{0} / r_{0}>i / r$. Hence $a^{r} y^{i} \in A$. Hence $a^{r} y^{s} \in A$; contradiction. Hence $s_{0} / r_{0} \leq s / r$.

Let $z=a^{r_{0}} y^{s_{0}}$. We have $I_{y} \subset I_{z}$, so by the maximality of $I_{y}$ we have $I_{y}=I_{z}$. For any $k \in \mathbb{N}$ we have $a z^{k}=a^{r_{0} k+1} y^{s_{0} k}$, and

$$
\frac{s_{0} k}{r_{0} k+1}<\frac{s_{0}}{r_{0}} \text {. }
$$

Hence $a z^{k} \in A$. Hence $a \in I_{z}$. Hence $a \in I_{y}$; contradiction. Hence $a y \notin A$.

Note that ay satisfies a monic polynomial of degree $n$ with coefficients in $A$. We have:

$$
I_{y} \subset\left[I_{y}: a\right] \subset\left[A: a y,(a y)^{2}, \ldots,(a y)^{n-1}\right]=I_{a y},
$$

so by the maximality of $I_{y}$ we must have $I_{y}=I_{a y}$, and hence $I_{y}$ is prime.

Let us say that an abelian group $G$ is pseudo-X-linear if there exists a filtration

$$
0=G_{0} \subset G_{1} \subset \cdots \subset G_{m}=G,
$$

with the property that $G_{i} / G_{i-1}$ is $X$-linear for each $i$. It is conceivable that every such group $G$ is $X$-linear.

Theorem 9.4. Assume that $X$ is reduced, and that the canonical map $\pi: X_{\text {nor }} \rightarrow$ $X$ is finite. Let $C$ be the quotient sheaf $\pi_{*} \mathcal{O}_{X_{\text {nor }}}^{*} / \mathcal{O}_{X}^{*}$. Then the group $C(X)$ is pseudo-X-linear. If $X$ is of finite type over $\mathbb{Z}$, then $C(X)$ is arithmetically linear.

Proof. The comment about what happens when $X$ is of finite type over $\mathbb{Z}$ is left to the reader; the proof given below works with appropriate changes, provided that one uses in addition (8.3)(b) and (8.5), and one uses (8.1) instead of (7.1).

The following two facts are easily verified: any subgroup of a pseudo- $X$-linear abelian group is pseudo- $X$-linear, and any product of finitely many pseudo- $X$-linear abelian groups is pseudo- $X$-linear. It follows that we may reduce to the case where $X$ is affine. Then by (9.3), we may reduce to the following situation: $A$ and $A^{\prime}$ are reduced noetherian rings, with $A \subset A^{\prime} \subset A_{\text {nor }}$ (and $A_{\text {nor }}$ is module-finite over $A$ ), and the ideal $\left[A: A^{\prime}\right]$ of $A$ is prime. We must show that if $F$ is the Zariski sheaf associated to the $A$-functor given by $B \mapsto\left(A^{\prime} \otimes_{A} B\right)^{*} / B^{*}$, then $F(A)$ is $X$-linear. But $F(A) \cong\left[\underline{\operatorname{PGL}}\left(A^{\prime}\right)\right](A)$, so by $(9.2)$, it suffices to show that if $G=\mathbf{A u t}_{\text {alg }}\left(\operatorname{End}\left(A^{\prime}\right)\right)$, then $G(A)$ is $X$-linear. But $G$ is coherent by (4.13), so the theorem follows from (7.1).

One can check that (7.2) applies to a pseudo- $X$-linear abelian group, so one obtains the following corollary:

Corollary 9.5. Assume that $X$ is reduced, and that the canonical map $\pi: X_{\text {nor }} \rightarrow$ $X$ is finite. Let $C$ be the quotient sheaf $\pi_{*} \mathcal{O}_{X_{\text {nor }}}^{*} / \mathcal{O}_{X}^{*}$. Then:

(a) There are only finitely many prime numbers $p$ such that ${ }_{p} C(X)$ is infinite. Moreover, such a $p$ cannot be invertible in $\Gamma\left(X, \mathcal{O}_{X}\right)$. 
(b) If $X$ is essentially of finite type over $\mathbb{Z}$ or $\mathbb{Z}_{p}$ (for some prime number $p$ ), then there exist prime numbers $p_{1}, \ldots, p_{n}$, none of which are invertible in $\Gamma\left(X, \mathcal{O}_{X}\right)$, such that the subgroup of $C(X)$ consisting of torsion prime to $p_{1} \cdots p_{n}$ is finite.

Lemma 9.6. Assume that $X$ is reduced, and that the canonical map $\pi: X_{\text {nor }} \rightarrow X$ is finite. Let $Q=\Gamma\left(\mathcal{O}_{X_{\text {nor }}}^{*}\right) / \Gamma\left(\mathcal{O}_{X}^{*}\right)$. Let $K$ be the kernel of the canonical map $\operatorname{Pic}(X) \rightarrow \operatorname{Pic}\left(X_{\text {nor }}\right)$. Let $C$ be the quotient sheaf $\pi_{*} \mathcal{O}_{X_{\text {nor }}}^{*} / \mathcal{O}_{X}^{*}$. Then there is a short exact sequence

$$
0 \rightarrow Q \rightarrow C(X) \rightarrow K \rightarrow 0 .
$$

Proof. Any finitely generated projective module of constant rank over a semilocal ring is free, so any line bundle on $X_{\text {nor }}$ may be trivialized by an open cover pulled back from $X$. (This argument was shown to me by R. Wiegand.) Hence $R^{1} \pi_{*}\left(\mathcal{O}_{X_{\text {nor }}}^{*}\right)=0$, and so by the Leray spectral sequence we see that $H^{1}\left(X, \pi_{*} \mathcal{O}_{X_{\text {nor }}}^{*}\right)$ $\cong \operatorname{Pic}\left(X_{\text {nor }}\right)$. Run the long exact sequence of cohomology on

$$
0 \rightarrow \mathcal{O}_{X}^{*} \rightarrow \pi_{*} \mathcal{O}_{X_{\text {nor }}}^{*} \rightarrow C \rightarrow 0
$$

to get the claimed exact sequence.

Corollary 9.7. Assume that $X$ is reduced, and that the canonical map $\pi: X_{\text {nor }} \rightarrow$ $X$ is finite. Let $Q=\Gamma\left(\mathcal{O}_{X_{\text {nor }}}^{*}\right) / \Gamma\left(\mathcal{O}_{X}^{*}\right)$. Let $K$ be the kernel of the canonical map $\operatorname{Pic}(X) \rightarrow \operatorname{Pic}\left(X_{\text {nor }}\right)$.

(a) Fix $n \in \mathbb{N}$, and assume that $n$ is invertible in $\Gamma\left(X, \mathcal{O}_{X}\right)$. If $Q$ is finitely generated, or more generally if it admits an $n$-divisible subgroup with finitely generated cokernel, then ${ }_{n} K$ is finite.

(b) If $Q$ admits a divisible subgroup with finitely generated cokernel, then there are only finitely many prime numbers $p$ such that ${ }_{p} K$ is infinite.

Proof. Apply the lemma. By (9.5), it suffices to show that if

$$
0 \rightarrow M^{\prime} \rightarrow M \rightarrow M^{\prime \prime} \rightarrow 0
$$

is a short exact sequence of abelian groups, $\left|{ }_{n} M\right|<\infty$, and there exists an $n$ divisible subgroup $H \subset M^{\prime}$ such that $M^{\prime} / H$ is finitely generated, then $\left|{ }_{n} M^{\prime \prime}\right|<\infty$. This is easily proved - see [20].

Example 9.8. Let $X=\operatorname{Spec} \mathbb{Q}[x, y] /\left(x^{2}-2 y^{2}\right)$. Then

$$
\Gamma\left(\mathcal{O}_{X_{\text {nor }}}^{*}\right) / \Gamma\left(\mathcal{O}_{X}^{*}\right) \cong \mathbb{Q}[\sqrt{2}]^{*} / \mathbb{Q}^{*},
$$

which is not finitely generated.

We consider what happens when $X$ is of finite type over $\mathbb{Z}$. We need the following well-known result, which is apparently due to Roquette. A proof of the key case ( $X$ integral, affine) may be found in ([7], p. 39).

Proposition 9.9. Let $X$ be a reduced scheme of finite type over $\mathbb{Z}$. Then the group $\Gamma\left(X, \mathcal{O}_{X}\right)^{*}$ is finitely generated.

Theorem 9.10. Let $X$ be a reduced scheme of finite type over $\mathbb{Z}$. Then the torsion subgroup of $\operatorname{Pic}(X)$ is supported at a finite set of primes, and if ${ }_{p} \operatorname{Pic}(X)$ is infinite, then the prime $p$ is not invertible in $\Gamma\left(X, \mathcal{O}_{X}\right)$. 
Proof. By ([24], 2.7.6) we know that $\operatorname{Pic}\left(X_{\text {nor }}\right)$ is finitely generated. Therefore it suffices to show that if $K=\operatorname{Ker}\left[\operatorname{Pic}(X) \rightarrow \operatorname{Pic}\left(X_{\text {nor }}\right)\right]$, then $K$ is supported at a finite set of primes, and if ${ }_{p} K$ is infinite, then the prime $p$ is not invertible in $\Gamma\left(X, \mathcal{O}_{X}\right)$.

By (9.4), (9.9) and the argument of (9.7), one sees that $K$ is isomorphic to the quotient of an arithmetically linear group by a finitely generated subgroup. Hence (8.15) tells us that the torsion subgroup of $\operatorname{Pic}(X)$ is supported at a finite set of primes. The last assertion of the theorem follows from (9.7)(a).

For $X$ a nonreduced scheme of finite type over $\mathbb{Z}$, we do not know if the torsion subgroup of $\operatorname{Pic}(X)$ is supported at a finite set of primes. However, for any commutative noetherian ring $A$, the canonical map $\operatorname{Pic}(A) \rightarrow \operatorname{Pic}\left(A_{\text {red }}\right)$ is an isomorphism, so we have:

Corollary 9.11. Let $A$ be a finitely generated commutative $\mathbb{Z}$-algebra. Then the torsion subgroup of $\operatorname{Pic}(A)$ is supported at a finite set of primes, and if $p \operatorname{Pic}(A)$ is infinite, then the prime $p$ is not invertible in $A$.

Let $A$ be a finitely generated commutative $\mathbb{Z}$-algebra. It is natural to ask if there exist prime numbers $p_{1}, \ldots, p_{n}$, none of which are invertible in $A$, such that the subgroup of $\operatorname{Pic}(A)$ consisting of torsion prime to $p_{1}, \ldots, p_{n}$ is finite. Unfortunately, the answer is no. For a counterexample, see ([20], 6.3).

Now we consider what happens when $X$ is of finite type over $\mathbb{F}_{p}$.

Theorem 9.12. Let $X$ be a scheme of finite type over $\mathbb{F}_{p}$. Then there exist a finitely generated abelian group $H$ and a finite p-group $F$ such that

$$
\operatorname{Pic}(X) \cong H \oplus\left[\bigoplus_{n=1}^{\infty} F\right] .
$$

Proof (Sketch). Consider the class $\mathcal{C}$ of groups of the form ascribed to $\operatorname{Pic}(X)$ in the theorem. The groups in $\mathcal{C}$ are all bounded $\times$ cobounded. One can check without great difficulty that $\mathcal{C}$ is closed under formation of subgroups, quotient groups, and extensions.

First suppose that $X$ is reduced. Since $\operatorname{Pic}\left(X_{\text {nor }}\right)$ is finitely generated, it suffices to show that

$$
K=\operatorname{Ker}\left[\operatorname{Pic}(X) \rightarrow \operatorname{Pic}\left(X_{\text {nor }}\right)\right]
$$

is in $\mathcal{C}$. We may assume that $X$ is affine. In fact, it suffices to show that if $X^{\prime}=\operatorname{Spec}\left(A^{\prime}\right)$ is a partial normalization of $X=\operatorname{Spec}(A)$, and if $\left[A^{\prime}: A\right]$ is prime, then $K^{\prime}=\operatorname{Ker}\left[\operatorname{Pic}(X) \rightarrow \operatorname{Pic}\left(X^{\prime}\right)\right]$ is in $\mathcal{C}$. The proof of $(9.10)$ shows that $K^{\prime}$ is a quotient of an arithmetically linear group. In fact, the arguments used to arrive at this result show that there exists an $\mathbb{F}_{p}$-algebra $A$ of finite type such that $K^{\prime}$ is a quotient of an abelian subgroup $H \subset \mathrm{GL}_{n}(A)$. Therefore there exists an $\mathbb{F}_{p}$-algebra $C$ of finite type such that $K^{\prime}$ is a quotient of a subgroup of $C^{*}$. The usual methods show that $C^{*}$ may be built up via extensions from a finitely generated abelian group and some $\mathbb{F}_{p}$-vector spaces. It follows that $C^{*} \in \mathcal{C}$, and hence that any quotient of $C^{*}$ is in $\mathcal{C}$.

Now suppose that $X$ is arbitrary, not necessarily reduced. Let $\mathcal{I}$ be the nilradical of $X$. There is an exact sequence

$$
0 \rightarrow \mathcal{I}^{n} / \mathcal{I}^{n+1} \rightarrow\left(\mathcal{O}_{X} / \mathcal{I}^{n+1}\right)^{*} \rightarrow\left(\mathcal{O}_{X} / \mathcal{I}^{n}\right)^{*} \rightarrow 1
$$


of sheaves of abelian groups on $X$. Let $X_{n}$ be the closed subscheme of $X$ corresponding to the ideal $\mathcal{I}^{n}$. By taking cohomology, one sees that

$$
\operatorname{Ker}\left[\operatorname{Pic}\left(X_{n+1}\right) \rightarrow \operatorname{Pic}\left(X_{n}\right)\right]
$$

is $p$-torsion. The theorem follows.

Finally, we compute the Picard group of a few simple examples (see Table 1). The calculations are an easy consequence of an exact sequence of Milnor ([6], IX 5.3) applied to the cartesian square

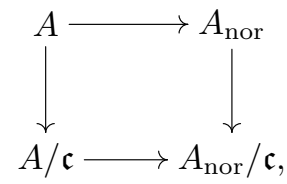

where $\mathfrak{c}$ is the conductor of $A_{\text {nor }}$ into $A$. The exact sequence is:

$$
\begin{aligned}
A^{*} & \rightarrow A_{\text {nor }}^{*} \times(A / \mathfrak{c})^{*} \rightarrow\left(A_{\text {nor }} / \mathfrak{c}\right)^{*} \\
& \rightarrow \operatorname{Pic}(A) \rightarrow \operatorname{Pic}\left(A_{\text {nor }}\right) \times \operatorname{Pic}(A / \mathfrak{c}) \rightarrow \operatorname{Pic}\left(A_{\text {nor }} / \mathfrak{c}\right) .
\end{aligned}
$$

Let $p$ be a prime number. The first four rings given in Table 1 may be viewed as subrings of $\mathbb{Z}[t, x]$.

\section{TABLE 1}

\begin{tabular}{||c|c||}
\hline $\operatorname{ring} A$ & structure of $\operatorname{Pic}(A)$ \\
\hline \hline $\mathbb{Z}\left[t^{2}, t^{3}\right]$ & $\mathbb{Z}$ \\
\hline $\mathbb{Z}\left[t^{2}, t^{3}, x\right]$ & free abelian of countably infinite rank \\
\hline $\mathbb{Z}\left[p t, t^{2}, t^{3}\right]$ & $\mathbb{F}_{p}$ \\
\hline $\mathbb{Z}\left[p t, t^{2}, t^{3}, x\right]$ & $\mathbb{F}_{p}$-vector space of countably infinite rank \\
\hline $\mathbb{Z}\left[1 / p, t^{2}, t^{3}\right]$ & $\mathbb{Z}[1 / p]$ \\
\hline $\mathbb{F}_{p}\left[t^{2}, t^{3}, x\right]$ & $\mathbb{F}_{p}$-vector space of countably infinite rank \\
\hline
\end{tabular}

\section{Appendix.}

\section{COHERENCE OF HIGHER DIRECT IMAGES AS FUNCTORS}

We consider a question which was posed (in an equivalent form) by Artin [2]:

Problem. Let $X$ be a proper $A$-scheme, let $\mathcal{F}$ be a coherent sheaf on $X$, and fix $n \geq 0$. Is the A-functor $H=H_{\mathcal{F}}^{n}$ given by $B \mapsto H^{n}\left(X_{B}, \mathcal{F}_{B}\right)$ module-coherent?

Taking the Čech resolution of $\mathcal{F}$ relative to some affine open cover of $X$ yields a complex $K$ of $A$-modules, and by $([16], 1.4 .1)$ we have $H^{n}\left(X_{B}, \mathcal{F}_{B}\right) \cong H^{n}(K \otimes B)$ for all commutative $A$-algebras $B$. It follows that at least $H$ is module-quasicoherent. The issue of whether $H$ is module-coherent is quite subtle. We will show that if $\mathcal{F}$ is $A$-flat, or $A$ is a Dedekind domain, then $H$ is module-coherent. By (6.10), we know also that $H$ is module-coherent if and only if $H$ preserves products, but it is not clear how to use this statement. We will give an example which shows that in general $H$ is not module-coherent.

Let us say that a module-valued $A$-functor $F$ is upper semicontinuous if for every commutative $A$-algebra $B$, and every $\mathfrak{p} \in \operatorname{Spec}(B)$, there is a neighborhood $U$ of 
$\mathfrak{p}$ such that $\operatorname{dim}_{k(\mathfrak{q})} F(k(\mathfrak{q})) \leq \operatorname{dim}_{k(\mathfrak{p})} F(k(\mathfrak{p}))$ for all $\mathfrak{q} \in U$. Similarly, one defines lower semicontinuous by reversing the inequality. If $M$ is a finitely generated $A$ module, then it follows by Nakayama's lemma that $\underline{M}$ is upper semicontinuous. If $f: P_{1} \rightarrow P_{2}$ is a map of finitely generated projective $A$-modules, then $\underline{\operatorname{Ker}}(f)$ is upper semicontinuous, whereas $\underline{\operatorname{Im}}(f)$ is lower semicontinuous. For $I \subset A$ an ideal, $\underline{\operatorname{Ker}}(A \rightarrow A / I)$ is in general not upper semicontinuous. (But it is lower semicontinuous.) If the numerator of a quotient is upper semicontinuous and the denominator is lower semicontinuous, then the quotient is itself upper semicontinuous. It follows that if $K_{0}$ is a complex of finitely generated free $A$-modules, and $n \in \mathbb{Z}$, then the module-valued $A$-functor given by $B \mapsto H^{n}\left(K_{0} \otimes B\right)$ is upper semicontinuous. It is also module-coherent.

Proposition 10.1. If $X$ is proper over $A$ and $\mathcal{F}$ is a coherent sheaf on $X$ which is A-flat, then the module-valued A-functor $H_{\mathcal{F}}^{n}$ given by $B \mapsto H^{n}\left(X_{B}, \mathcal{F}_{B}\right)$ is module-coherent and upper semicontinuous.

Proof (pointed out to me by Deligne and Ogus; cf. [22]). Let $K$ be the Čech complex discussed above. It is bounded and flat. Since $X$ is proper over $A$, the complex $K$ has finitely generated cohomology modules. It follows that there exist a complex $K_{0}$ of finitely generated free $A$-modules which is bounded above, and a quasiisomorphism $\phi: K_{0} \rightarrow K$. Since $K$ is flat and bounded above, there is a spectral sequence

$$
E_{p, q}^{2}=\operatorname{Tor}_{p}\left(H^{-q}(K), B\right) \Rightarrow H^{-p-q}(K \otimes B) .
$$

(See, for example, [27], 11.34). Similarly, one has such a spectral sequence for $K_{0}$. Moreover, $\phi$ induces a morphism from the spectral sequence for $K_{0}$ to the spectral sequence for $K$. Since $\phi$ is a quasi-isomorphism, the induced maps $H^{-q}\left(K_{0}\right) \rightarrow$ $H^{-q}(K)$ are isomorphisms, and so the induced maps

$$
\operatorname{Tor}_{p}\left(H^{-q}\left(K_{0}\right), B\right) \rightarrow \operatorname{Tor}_{p}\left(H^{-q}(K), B\right)
$$

are isomorphisms. Hence $H^{n}(\phi)$ is an isomorphism. Hence the module-valued $A$-functor $B \mapsto H^{n}\left(X_{B}, \mathcal{F}_{B}\right)$ is isomorphic to the module-valued $A$-functor $B \mapsto$ $H^{n}\left(K_{0} \otimes B\right)$.

The upper semicontinuity part of the proposition is of course the usual theorem on upper semicontinuity of cohomology (see, e.g., [21], III, 12.8).

Theorem 10.2. If $X$ is proper over a Dedekind domain $A$ and $\mathcal{F}$ is a coherent sheaf on $X$, then the module-valued $A$-functor $H_{\mathcal{F}}^{n}$ given by $B \mapsto H^{n}\left(X_{B}, \mathcal{F}_{B}\right)$ is module-coherent.

Before proving this, there are some preliminaries. By a truncated discrete valuation ring, we shall mean a ring $A$ of the form $R / I$ where $R$ is a discrete valuation ring and $I$ is a proper nonzero ideal. By a uniformizing parameter for $A$, we shall mean the image in $A$ of a uniformizing parameter for $R$.

Lemma 10.3. Let $A$ be a truncated discrete valuation ring with uniformizing parameter $t$. Let $C$ be a bounded complex of $A$-modules. Assume that $H^{n}\left(C \otimes A /\left(t^{l}\right)\right)$ is finitely generated for all $n$ and all $l$. Then for each $n$, the $A$-functor $F$ given by $B \mapsto H^{n}(C \otimes B)$ is module-coherent.

Proof. Any module $M$ over $A$ is a direct sum of cyclic modules. (See, e.g., [10], Chapter VII, §2, Exercise 12(b).) It follows that if $M_{0}$ is a finitely generated 
submodule of $M$, then there exists a finitely generated direct summand $M_{1}$ of $M$ with $M_{0} \subset M_{1}$.

For each $l$, let $A_{l}$ denote $A /\left(t^{l}\right)$. By working from low indices to high indices, one can construct a subcomplex $C_{0}$ of $C$ with the properties that for each $n$ :

(a) for each $l, \operatorname{Ker}\left[d^{n} \otimes A_{l}\right] \subset C_{0}^{n} \otimes A_{l}+\operatorname{Im}\left[d^{n-1} \otimes A_{l}\right]$, and

(b) $C_{0}^{n}$ is finitely generated and is a direct summand of $C^{n}$.

Property (b) comes from the first paragraph.

Let $\bar{C}=C / C_{0}$. It follows from (b) that the sequence

$$
0 \rightarrow C_{0} \rightarrow C \rightarrow \bar{C} \rightarrow 0
$$

is universally exact. Since any $A$-module is a direct sum of cyclic modules, it follows from (a) that the induced map $H^{n}\left(C_{0} \otimes B\right) \rightarrow H^{n}(C \otimes B)$ is surjective for every $n$ and every $B$, and hence that we have short exact sequences

$$
0 \rightarrow H^{n-1}(\bar{C} \otimes B) \rightarrow H^{n}\left(C_{0} \otimes B\right) \rightarrow H^{n}(C \otimes B) \rightarrow 0 .
$$

Now construct a subcomplex $\bar{C}_{0} \subset \bar{C}$ in the same way that we constructed $C_{0} \subset C$. It follows that $F$ is expressible as the cokernel of a map of module-coherent functors, and hence that $F$ is itself module-coherent.

Now we prove (10.2), using arguments provided by Deligne.

Proof of (10.2). There is an exact sequence of coherent sheaves on $X$,

$$
0 \rightarrow \mathcal{F}^{\prime} \rightarrow \mathcal{F} \rightarrow \mathcal{F}^{\prime \prime} \rightarrow 0
$$

in which $\mathcal{F}^{\prime}$ is $A$-torsion and $\mathcal{F}^{\prime \prime}$ is $A$-torsion-free. Since $A$ is a Dedekind domain, $\mathcal{F}^{\prime \prime}$ is $A$-flat. Hence this sequence remains exact after tensoring over $A$ by anything, so by the long exact sequence of cohomology and (5.15), it suffices to prove the theorem when $\mathcal{F}$ is either $A$-torsion or $A$-flat. The second case is taken care of by (10.1). Therefore we may assume that $\mathcal{F}$ is $A$-torsion. Then in fact $a \mathcal{F}=0$ for some nonzero $a \in A$. We may then reduce to the case where $A$ is a truncated discrete valuation ring. Apply (10.3) to the Čech complex of $\mathcal{F}$.

Finally we give a counterexample which shows that, in general, $H_{\mathcal{F}}^{n}$ is not modulecoherent. The counterexample to be given here is based on examples constructed by H. Cohen [12]. The same examples appear in his thesis [13], where Cohen remarks briefly that the techniques therein yield a counterexample to Artin's problem. However, he says nothing more about the matter. It seems likely that Cohen and/or Verdier did construct a counterexample, but it has now (apparently) been lost.

Let $k$ be a field, let $A=k[[s, t]]$, and let $X=\mathbb{P}_{A}^{3}$, with coordinates $x, y, z, w$. Let $\mathcal{F}$ be the cokernel of the map $\mathcal{O}_{X} \rightarrow \mathcal{O}_{X}(1)$ given by multiplication by $s x-t y .{ }^{4}$ Let $n=1$. We will show that $H$ is not module-coherent, using (4.2).

In Proposition 2 of [12], Cohen shows (in effect) that if $B$ is a commutative $A$-algebra, and $R=B\left[x^{ \pm 1}, y^{ \pm 1}, z^{ \pm 1}, w^{ \pm 1}\right]$, then $H(B)$ is isomorphic to the degree 0 part of the quotient of

$$
\left\{\frac{f}{x^{a} y^{b} z^{c} w^{d}} \in R: f \in B[x, y, z, w], a, b, c, d \in \mathbb{N}, \text { and }(s x-t y) f=0\right\}
$$

\footnotetext{
${ }^{4}$ In terms of Cohen's construction, we have $r=3, c_{0}=s, c_{1}=-t$, and $m=0$.
} 
by the sub- $B[x, y, z, w]$-module generated by

$$
\left\{\frac{f}{y z w}, \frac{f}{x z w}, \frac{f}{x y w}, \frac{f}{x y z} \in R: f \in B[x, y, z, w] \text { and }(s x-t y) f=0\right\} .
$$

Let $B=A /\left(s^{k}, t^{k}\right)$, for some $k \in \mathbb{N}$. We proceed to compute $H(B)$.

Lemma 10.4. The $B[x, y, z, w]$-module $\operatorname{Ann}_{B[x, y, z, w]}(s x-t y)$ is generated by

$$
(s t)^{k-1},(s t)^{k-2} \sum_{i=0}^{1}(s x)^{i}(t y)^{1-i}, \ldots,(s t)^{0} \sum_{i=0}^{k-1}(s x)^{i}(t y)^{k-1-i} .
$$

Proof (Sketch). Let $C=k[s, t, x, y] /\left(s^{k}, t^{k}\right)$. Since $B[x, y, z, w]$ is a flat $C$-algebra, it suffices to show that the $C$-module $\operatorname{Ann}_{C}(s x-t y)$ admits the given generators. Let $f \in \operatorname{Ann}_{C}(s x-t y)$. Write

$$
f=\sum_{0 \leq i, j \leq k-1} f_{i j} s^{i} t^{j}
$$

where $f_{i j} \in k[x, y]$. The following assertions are easily checked:

- $f_{i, 0}=0$ if $0 \leq i \leq k-2$;

- $f_{0, j}=0$ if $0 \leq j \leq k-2$;

- $f_{i, j-1}=(x / y) f_{i-1, j}$ if $1 \leq i, j \leq k-1$.

Hence $f$ is completely determined by $f_{0, k-1}, \ldots, f_{k-1, k-1}$. The lemma follows.

From this lemma it follows that $H(B)$ is isomorphic to the sub- $B$-module of $R$ generated by

$$
\bigcup_{j=5}^{k}\left\{\frac{(s t)^{k-j} \sum_{i=0}^{j-1}(s x)^{i}(t y)^{j-1-i}}{x^{a} y^{b} z^{c} w^{d}}: a, b, c, d \in \mathbb{N} \text { and } a+b+c+d=j-1\right\} .
$$

Since this is a minimal generating set, we have

$$
\mu[H(B)]=\sum_{j=5}^{k}\left(\begin{array}{c}
j-2 \\
3
\end{array}\right) \geq O\left(k^{3}\right)
$$

Since $(s, t)^{2 k-1} \subset\left(s^{k}, t^{k}\right)$, it follows from (4.2) that $H$ is not module-coherent.

\section{REFERENCES}

[1] M. Artin, Algebraic approximation of structures over complete local rings, Inst. Hautes Études Sci. Publ. Math. 36 (1969), 23-58. MR 42:3087

[2] L Letter to Grothendieck, Nov. 5, 1968.

[3] - Algebraization of formal moduli. I, Global Analysis: Papers in Honor of K. Kodaira, Princeton Univ. Press, Princeton, N.J., and Univ. of Tokyo Press, Tokyo, 1969, pp. 21-71. MR 41:5369

[4] M. F. Atiyah and I. G. Macdonald, Introduction to Commutative Algebra, Addison-Wesley, Reading, MA, 1969. MR 39:4129

[5] M. Auslander, Coherent functors, Proc. Conf. Categorical Algebra (La Jolla, 1965; S. Eilenberg et al., eds.), Springer-Verlag, New York, 1966, pp. 189-231. MR 35:2945

[6] H. Bass, Algebraic K-Theory, Benjamin, New York, 1968. MR 40:2736

[7] Introduction to some methods of algebraic K-theory, Conf. Board Math. Sci. Regional Conf. Ser. Math., no. 20, Amer. Math. Soc., Providence, RI, 1974.

[8] G. M. Bergman, Ring schemes: the Witt scheme, in D. Mumford, Lectures on Curves on an Algebraic Surface, Princeton Univ. Press, Princeton, NJ, 1966, pp. 171-187. MR 35:187 
[9] J. E. Bertin, Généralités sur les préschémas en groupes, Séminaire de Géométrie Algébrique du Bous-Marie 1963/64 (SGA 3), Lecture Notes in Math. 151, Springer-Verlag, New York, 1970, pp. 318-410. MR 38:3275

[10] N. Bourbaki, Algebra, II. Chapters 4-7, Springer-Verlag, New York, 1990. MR 91h:00003

[11] L. Claborn, Every abelian group is a class group, Pacific J. Math. 18 (1966), 219-222. MR 33:4085

[12] H. Cohen, Un faisceau qui ne peut pas être détordu universellement, C. R. Acad. Sci. Paris Sér. A 272 (1971), 799-802. MR 44:5322

[13] _ Détorsion universelle de faisceaux cohérents, thesis (Docteur $3^{\circ}$ Cycle), Univ. ParisXI (Orsay), 1972.

[14] R. M. Fossum, The Divisor Class Group of a Krull Domain, Springer-Verlag, New York, 1973. MR 52:3139

[15] L. Fuchs, Abelian Groups, Publ. House Hungarian Acad. Sci., Budapest, 1958. MR 21:5672

[16] A. Grothendieck and J. A. Dieudonné, Éléments de géométrie algébrique. III (part one), Inst. Hautes Études Sci. Publ. Math. 11 (1961). MR 29:1209

[17] Éléments de géométrie algébrique. III (part two), Inst. Hautes Études Sci. Publ. Math. 17 (1963). MR 29:1210

[18] Éléments de géométrie algébrique. IV (part three), Inst. Hautes Études Sci. Publ. Math. 28 (1966).

[19] _ Éléments de Géométrie Algébrique. I, 2nd ed., Springer-Verlag, New York, 1971. Zbl. 203, 233.

[20] R. Guralnick, D. B. Jaffe, W. Raskind and R. Wiegand, On the Picard group: torsion and the kernel induced by a faithfully flat map, J. Algebra (to appear).

[21] R. Hartshorne, Algebraic Geometry, Springer-Verlag, New York, 1977. MR 57:3116

[22] L. Illusie, Généralités sur les conditions de finitude dans les catégories dérivées, Séminaire de Géométrie Algébrique du Bois-Maris 1966/67 (SGA 6), Lecture Notes in Math. 225, Springer-Verlag, New York, 1971, pp. 78-159. MR 50:7133

[23] N. Koblitz, p-adic Numbers, p-adic Analysis, and Zeta-Functions, Springer-Verlag, New York, 1977. MR 57:5964

[24] S. Lang, Fundamentals of Diophantine Geometry, Springer-Verlag, New York, 1983. MR 85j: 11005

[25] _ Algebra, 2nd ed., Addison-Wesley, Reading, MA, 1984. MR 86j:00003

[26] S. Mac Lane, Categories for the Working Mathematician, Springer-Verlag, New York, 1971. MR 50:7275

[27] J. J. Rotman, An Introduction to Homological Algebra, Academic Press, New York, 1979. MR 80k: 18001

[28] J.-P. Serre, Local Fields, Springer-Verlag, New York, 1979. MR 82e:12016

Department of Mathematics and Statistics, University of Nebraska, Lincoln, NeBRASKA 68588-0323

E-mail address: jaffe@cpthree.unl.edu 